\title{
The Role of Small Scale Experiments in the Direct Detection of Dark Matter
}

\author{
Susana Cebrián ${ }^{1,2}$ (D) \\ 1 Centro de Astropartículas y Física de Altas Energías (CAPA), Universidad de Zaragoza, C/ Pedro Cerbuna \\ 12, 50009 Zaragoza, Spain; scebrian@unizar.es \\ 2 Laboratorio Subterráneo de Canfranc, Paseo de los Ayerbe s/n, 22880 Canfranc Estación, Spain
}

Citation: Cebrián, S. The Role of Small Scale Experiments in the Direct Detection of Dark Matter. Universe 2021, 7, 81. https://doi.org/10.3390/ universe7040081

Academic Editors: Ivan De Martino and Panayiotis Stavrinos

Received: 26 February 2021

Accepted: 25 March 2021

Published: 28 March 2021

Publisher's Note: MDPI stays neutral with regard to jurisdictional claims in published maps and institutional affiliations.

Copyright: (c) 2021 by the authors. Licensee MDPI, Basel, Switzerland. This article is an open access article distributed under the terms and conditions of the Creative Commons Attribution (CC BY) license (https:// creativecommons.org/licenses/by/ $4.0 /)$.

\begin{abstract}
In the direct detection of the galactic dark matter, experiments using cryogenic solid-state detectors or noble liquids play for years a very relevant role, with increasing target mass and more and more complex detection systems. But smaller projects, based on very sensitive, advanced detectors following new technologies, could help in the exploration of the different proposed dark matter scenarios too. There are experiments focused on the observation of distinctive signatures of dark matter, like an annual modulation of the interaction rates or the directionality of the signal; other ones are intended to specifically investigate low mass dark matter candidates or particular interactions. For this kind of dark matter experiments at small scale, the physics case will be discussed and selected projects will be described, summarizing the basics of their detection methods and presenting their present status, recent results and prospects.
\end{abstract}

Keywords: dark matter; direct detection; annual modulation; directionality; low mass dark matter

\section{Introduction}

According to a great deal of cosmological and astrophysical observations at different scales, there is a need of both dark energy and dark matter (DM) in the Universe; although the dark matter particles could explain an important fraction of the its energy-mass budget, its nature is unknown [1,2]. A plethora of candidates have been proposed to form the galactic DM, spanning over tens of orders of magnitude both in mass and interaction cross sections; axions and Weakly Interacting Massive Particles (WIMPs) are ones of the preferred candidates. Different approaches are followed in the investigation of the DM particles, based on the direct detection [3], indirect searches after annihilation [4] or production at accelerators [5].

The direct detection of DM was focused for years on the elastic scattering off target nuclei of WIMPs, for spin-independent (SI) and spin-dependent (SD) interactions, giving rise to nuclear recoils (NR) as detectable signals; but inelastic scattering and also scattering with electrons in the detector medium producing electron recoils (ER) are being considered too, as it will be shown later. Effective field theories (EFTs) including a minimal set of interactions and particles are being used in some cases as the frame to derive experimental results considering a number of operators describing the various possible interactions in a general way [6]. Assuming a particular type of interaction, the expected counting rates can be predicted considering certain astrophysical parameters (like the local density, the velocity distribution and the escape velocity of the DM particles) and properties of the DM particle [7]; the direct detection formalism is summarized, for example, in the Dark Matter section of [2] and detailed for elastic NR in [8].

This direct detection mechanism is really challenging as the produced signal is rare, with very low energy and has a continuum energy spectrum (which decays almost exponentially) making it indistinguishable from backgrounds [9]. The DM signal is searched in the detectors at the keV-scale and below and the expected interaction rates are typically 
lower than 1 event per $\mathrm{kg}$ and year. Therefore, the two basic requirements for this type of experiments are:

- Very low energy threshold.

- Ultra low background conditions. As for other rare event searches, operation deep underground to suppress cosmic rays and the use of passive and active shieldings against the environmental radiation from radioactivity and neutrons are mandatory $[10,11]$. Careful control of the material intrinsic radiopurity (in bulk or on surface) and of the cosmogenic activation of components producing long-lived radionuclides is also a must $[12,13]$. Appropriate materials must be selected based on different types of radioassays and purification techniques are often applied. The implementation of specific background rejection techniques, following for instance ER/NR discrimination by measuring different observables (heat, light and charge), Pulse Shape Discrimination (PSD) or volume fiducialisation if spatial information of events is available, has allowed to reduce the background levels in some experiments event down to $10^{-4} \mathrm{c} / \mathrm{keV} / \mathrm{kg} / \mathrm{d}$, leaving neutrinos (from the Sun, supernovae or the atmosphere) as an irreducible background [14].

The accumulation of a large quantity of target material and very long data taking periods expanding for months or years are also required, together with the stability of the environmental conditions and, in general, of the response of the detector along all that period. Additionally, the detection of distinctive signatures, such as an annual modulation in the rates of interaction or the signal directionality, would be priceless to attribute a DM origin to a potential observation.

Different types of experiments based on different, complementary technologies are underway, using for instance bolometers, liquid Argon or Xenon detectors, scintillators, semiconductors, gas detectors or bubble chambers $[9,15]$. Experiments based on bolometers and on noble liquid detectors with continuously increasing target mass have released in the last years the strongest limits on DM interaction cross sections following the direct detection approach. But initiatives for smaller projects based on different technologies are being now strongly supported by the community [16], as they can help to investigate well-motivated candidates, particularly for masses below the $\mathrm{GeV} / \mathrm{c}^{2}$ scale. The aim of this work is to review the goals and status of these other direct detection projects. The structure of the paper is the following. In the rest of this section, a brief summary of the latest results and prospects of experiments using liquid Argon and Xenon detectors and bolometers will be given for the sake of completeness (as they are not addressed more in the review). Running experiments and projects intended to identify distinctive signatures of the DM interaction will be presented in Section 2, for the annual modulation in the expected rates, and in Section 3, for the signal directionality. Those intended to specifically explore low mass DM using purely ionization detectors, like gas chambers or semiconductors, will be discussed in Section 4 while Section 5 will be devoted to experiments focused on SD interactions. Finally, summary and outlook will be given in Section 6 .

\subsection{Liquid Ar and Xe Detectors}

In noble gases, like Argon and Xenon, both scintillation and ionization are produced. They are being used in liquid as massive and compact DM targets, which are producing dominant results for high mass WIMPs, from a few $\mathrm{GeV} / \mathrm{c}^{2}$ to $\mathrm{TeV} / \mathrm{c}^{2}$. A dual-phase liquid noble gas detector is a Time Projection Chamber (TPC) which can register by means of adequate photosensors both the primary (S1) and the secondary (S2) scintillation from drifted electrons; then, the ratio S1/S2 can be used to discriminate ER from NR, with a threshold around $1 \mathrm{keV}_{e e}$ (Electron equivalent energy). Additionally, a 3D event reconstruction is possible with spatial resolution at the order of $\mathrm{mm}$ (using the coordinate deduced from the drift time). Targets at the level of tens, hundreds and even tons of $\mathrm{kg}$ have been already operated; the chamber is at the heart of the detection systems, accompanied by huge radiation shields, veto detectors, cryogenic and gas purification systems, elaborated data acquisition equipment and other ancillary systems. 
Three large dual-phase Xenon experiments have presented in the last years the leading constraints on SI DM-nucleus interactions for masses higher than $6 \mathrm{GeV} / \mathrm{c}^{2}$ : the XENON1T experiment at Laboratori Nazionali del Gran Sasso (LNGS), in Italy [17], LUX, already finished, at the Sanford Underground Research Facility (SURF), in US [18]; and the PANDAX-II experiment operated at Jinping Underground Laboratory, in China $[19,20]$. XMASS-I operates a single-phase liquid Xenon detector at the Kamioka Observatory in Japan [21]. Using only the S2 signal, results for DM-electron scattering have also been obtained by XENON1T with a threshold of $0.4 \mathrm{keV}_{e e}$ [22] and by PANDAX-II [23]. Specific investigation of light DM, being enhanced by the Migdal effect [24] or Bremsstrahlung when searching for ER, has been carried out by XENON1T [25] and by LUX [26]. In addition, a wide range of different DM models has been taken into account by the collaborations (effective interactions, inelastic scattering, light mediators, bosonic Super-WIMPs, dark photons ...); axions have been considered when looking for the origin of the ER excess reported by XENON1T [27]. The presence in natural Xenon of ${ }^{129} \mathrm{Xe}$ and ${ }^{131} \mathrm{Xe}$, with non-null spin, makes these detectors sensitive also to SD interactions. Extensions of the three dual-phase experiments, using tonnes of Xenon active mass at the same locations, are now being prepared or commissioned with expected operation along the next yearsXENONnT, LUX-ZEPLIN (LZ) and PANDAX-4T. The running of the DARWIN observatory in preparation, with 40 tonnes of Xenon, is foreseen for the end of the decade.

In Argon detectors, a very efficient PSD is possible for ER and NR. DEAP-3600, with a single-phase liquid Ar detector at SNOLAB in Canada, has demonstrated an excellent background rejection capability [28]. DarkSide-50 has operated at LNGS a dual-phase liquid Ar detector, filled with underground $\mathrm{Ar}$ (UAr) with strongly suppressed (by 1400) ${ }^{39} \mathrm{Ar}$ activity [29]; detecting the $\mathrm{S} 2$ signal only and achieving a $100 \mathrm{eV}_{e e}$ energy threshold, the search for low mass DM gave the strongest limits from 1.8 to $3.5 \mathrm{GeV} / \mathrm{c}^{2}$ for SI DMnucleus interaction [30] and also results on DM-electron interaction [31]. Effective field theory interactions have been also considered in the analysis of these Argon detectors. The Global Argon DM Collaboration (GADMC) has been constituted to cooperate for the procurement of radiopure UAr from the Urania plant in Colorado, US, and the Aria facilities in Sardinia, Italy, as well as for the development of SiPMs for readout. The DarkSide-20k detector will operate, as the first step, 20 tonnes of active mass of UAr inside a $3.5 \mathrm{~m}$ long TPC made with acrylic walls and placed inside an atmospheric Ar veto at LNGS; a smaller detector optimized for low mass DM investigation with just 1 tonne of purified UAr (DarkSide-LowMass) is also in preparation. In a longer term, the ARGO project, with 300 tonnes of $\mathrm{UAr}$, could be in operation at SNOLAB at the end of the decade; atmospheric neutrinos could be the limiting background.

\subsection{Bolometers}

In solid-state detectors operated at temperatures of tens of $\mathrm{mK}$ o even below, phonons (heat) can be registered by the very small increase of temperature induced; the simultaneous detection of scintillation (light) or ionization (charge) signals makes possible the discrimination between NR and ER. No quench affects the measured heat signal in these detectors. The operation temperature requires the use of powerful cryogenic systems to cool down even tens of $\mathrm{kg}$ of crystals. Very low energy thresholds below $100 \mathrm{eV}_{n r}$ (Nuclear recoil energy) have been reached, specially using crystals with very small mass; therefore, this kind of detectors are releasing the stringest results for DM-nucleus scattering in $\mathrm{GeV} / \mathrm{c}^{2}$ and sub-GeV $/ \mathrm{c}^{2}$ mass regions.

EDELWEISS-III presented very good bounds from 5 to $30 \mathrm{GeV} / \mathrm{c}^{2}$, and also results on Axion-Like Particles (ALPs) [32], operating 24 Germanium bolometers (each one with a mass of $870 \mathrm{~g}$ ) at the Laboratoire Souterrain de Modane (LSM), in France. EDELWEISS is now working with small germanium bolometers (with mass of $33 \mathrm{~g}$ ), first above ground and then at Modane, having reached a $60 \mathrm{eV}$ energy threshold. Masses down to $45 \mathrm{MeV} / \mathrm{c}^{2}$ when including the Migdal effect have been explored and DM-electron scattering as well as dark photons have been taken into account too [33,34]. 
SuperCDMS has operated Silicon and Germanium bolometers, also at the scale of hundreds of grams each, at the Soudan Underground Laboratory, in US. An energy threshold of $70 \mathrm{eV}$ was obtained, thanks to the NTL (Neganov-Trofimov-Luke) effect (applying high bias voltage to transform ionization to heat); results for DM-nucleus scattering up to $1.5 \mathrm{GeV} / \mathrm{c}^{2}$ and for both SI and SD interactions were obtained from different analyses [35,36]. Sensitivity to SD scattering is possible thanks to the presence of ${ }^{73} \mathrm{Ge}$. More recently, very small Silicon crystals (with masses of $0.93 \mathrm{~g}$ and $10.6 \mathrm{~g}$ ) have been operated on surface, deriving limits on DM-nucleon cross sections and also taking into account DM-electron interactions and dark photons $[37,38]$. The beginning of the operation at SNOLAB of SuperCDMS, combining different kinds of Silicon and Germanium detectors working in different modes with a total target mass of $\sim 30 \mathrm{~kg}$, is foreseen for 2021 .

Similarly, after using scintillating bolometers of $\mathrm{CaWO}_{4}$ with a mass of $300 \mathrm{~g}$ each in CRESST-II [39], ten $\mathrm{CaWO}_{4}$ crystals with a much smaller mass each (24 g) are being operated at LNGS in CRESST-III. An energy threshold of $30 \mathrm{eV}$ has been obtained, setting the best bounds for SI DM-nucleus interaction up to $160 \mathrm{MeV} / \mathrm{c}^{2}$ and similarly for SD interaction at low mass, particularly for the neutron-only case using also a $\mathrm{Li}_{2} \mathrm{MoO}_{4}$ crystal containing ${ }^{7} \mathrm{Li}$ and ${ }^{17} \mathrm{O}[40,41]$. In the next future, operation of about 100 crystals is planned and the energy threshold could be lowered down to $10 \mathrm{eV}$.

\section{Annual Modulation Effect}

As the Earth moves around the Sun, the relative velocity between the DM particles (within the galactic halo) and the detector (placed in the Earth) follows a cosine dependence; this gives rise to a modulation in time in the DM interaction rate, $S$, which can be expressed for an energy bin $i$ as:

$$
S_{i}(t)=S_{0, i}+S_{m, i} \cos \left(\omega\left(t-t_{0}\right)\right),
$$

being $S_{0, i}$ the non-modulated component and $S_{m, i}$ the modulation amplitude [42]. Assuming a locally isotropic DM halo, the modulation period $T=2 \pi / \omega$ is one year and the phase makes the maximum rate be expected around 2 June. The effect has well-defined properties: it must be weak (just a few percent variation), noticeable only at low energy and a phase reversal is expected at the very low energy region $[7,42,43]$. As no background component is known to show all these features, its identification is considered a distinctive signature of the DM interaction. It is worth noting that the features of the modulation effect are different for other DM distributions, for instance, in the case of sub-structures like streams and clumps or if considering a DM disc.

Scintillating crystals made of $\mathrm{NaI}(\mathrm{Tl})$ and coupled to photomultipliers (PMTs) are perfectly suited for the detection of the DM annual modulation, being robust and affordable detectors; they are capable of running in stable conditions for long times and a large target mass ca be easily accumulated. In any case, to achieve an ultra-low background (reducing significantly the content of ${ }^{210} \mathrm{~Pb}$ and ${ }^{40} \mathrm{~K}$ in commercial low-background $\mathrm{NaI}(\mathrm{Tl})$ detectors) and a low energy threshold, it has been necessary to implement new developments also in this type of conventional detectors.

The DAMA/LIBRA (DArk MAtter/Large sodium Iodide Bulk for RAre processes) experiment has been collecting data at LNGS for more than two decades. NaI(Tl) detectors fabricated by Saint Gobain company ( $9.7 \mathrm{~kg}$ each) are used [44]; nine units were firstly operated and, since 2003, there are 25 modules having a total mass of $\sim 250 \mathrm{~kg}$. All PMTs were replaced in 2011 for the second phase of the experiment, which allowed to reduce from 2 to $1 \mathrm{keV}_{e e}$ the software threshold. In the region of interest, the measured background level goes from 0.5 to $1 \mathrm{c} / \mathrm{keV} / \mathrm{kg} / \mathrm{d}$ [45]. The results from the first phase [46] were corroborated by the ones of the second one [45,47], reporting the presence of a modulation signal with all the expected properties at $12.9 \sigma$ C.L., following an exposure of 2.46 tonne $y$ collected over 20 years. The modulation amplitude deduced in the $2-6 \mathrm{keV}_{e e}$ window is $S_{m}=(0.0103 \pm 0.0008) \mathrm{c} / \mathrm{keV} / \mathrm{kg} / \mathrm{d}$; compatible results were obtained when applying other fitting methods and considering different periods of time, detector units and energy windows from 1 to $6 \mathrm{keV}_{e e}$. After the second phase of DAMA/LIBRA, new 
model-dependent, corollary analyses have been presented [47,48]; maximum likelihood procedures have been applied to define the allowed regions in the space of parameters of several scenarios, by comparing with the theoretical expectations the derived amplitude for the annual modulation. A third phase of the experiment is now in preparation, implementing hardware improvements with the aim to reduce the threshold below $1 \mathrm{keV}_{e e}$.

Considering the bounds on DM interaction cross sections presented by other direct detection experiments (see for instance the compilation at [2]), a tension appears if interpreting the DAMA/LIBRA modulation signal as due to DM, considering standard but also more general halo or interaction models. In experiments using different targets, annual modulation has not been observed neither with xenon [49-51] nor with germanium [52]. In this situation, to prove or disprove in a model-independent way the DAMA/LIBRA observation using the same target and detection technique would be essential. Various projects have undertaken this task; presently, only COSINE-100 and ANAIS-112 are already taking data.

The ANAIS experiment (Annual modulation with NAI Scintillators) operates at the Canfranc Underground Laboratory (LSC, "Laboratorio Subterráneo de Canfranc") in Spain; ANAIS-112 includes nine $\mathrm{NaI}(\mathrm{Tl})$ detectors $(112.5 \mathrm{~kg}$ in total) manufactured by the Alpha Spectra company [53]. The DM data taking is running since August 2017, with an excellent light collection at the level of $\sim 15 \mathrm{phe} / \mathrm{keV}$ for all detector units [54], which allows to work with an energy threshold at $1 \mathrm{keV}_{e e}$. A careful study of the different background components from the data taken with all the detectors, specific measurements and Monte Carlo simulations has been made [55-57] and discrimination methods for rejecting nonscintillation events are implemented [53]; from 1 to $6 \mathrm{keV}_{e e}$, the background level is measured as $3.6 \mathrm{c} / \mathrm{keV} / \mathrm{kg} / \mathrm{d}$ after efficiency correction for cuts [57]. The sensitivity of ANAIS-112 was assessed from this measured background, confirming the exploration of the $3 \sigma$ region singled out by DAMA/LIBRA from five years of data [58]. First results from an annual modulation analysis were published considering the first 1.5 years, corresponding to an exposure of $157.55 \mathrm{~kg} \cdot \mathrm{y}$ [59]. Updated results were presented for 2 years (with a total exposure of $220.69 \mathrm{~kg} \cdot \mathrm{y}$ ) applying the same analysis methods [60]. The analysis of the first 3 years of data (corresponding to $313.95 \mathrm{~kg} \cdot \mathrm{y}$ ) has been released, being the modulation amplitudes from best fits obtained $S_{m}=(-0.0034 \pm 0.0042) \mathrm{c} / \mathrm{keV} / \mathrm{kg} / \mathrm{d}$ for the $1-6 \mathrm{keV}_{e e}$ region and $S_{m}=(0.0003 \pm 0.0037) \mathrm{c} / \mathrm{keV} / \mathrm{kg} / \mathrm{d}$ in $2-6 \mathrm{keV}_{e e}$ [61]; this supports the absence of modulation in the data and the values are incompatible with DAMA/LIBRA result at 3.3 (2.6) $\sigma$ in the $1-6 \mathrm{keV}_{e e}\left(2-6 \mathrm{keV}_{e e}\right)$ windows, for a sensitivity of 2.5 (2.7) $\sigma$. Improvements in the background modelling for the fitting of the rates in the region of interest have been implemented in the analysis of the $3 \mathrm{y}$ data. Additionally, several consistency checks and some complementary analyses (a phase-free annual modulation search and the exploration of possible periodic signals at other frequencies) have been performed too at [61]. All the obtained results have allowed to fully confirm the expected sensitivity and the data taking is smoothly ongoing.

The COSINE project was conceived to join efforts between the DM-Ice experiment installed in the South Pole [62] and the KIMS collaboration. In COSINE-100, eight NaI(Tl) detectors (106 kg in total) built also by the Alpha Spectra company are operated at the Yangyang underground Laboratory in South Korea, being immersed in 22001 of liquid scintillator (to veto mainly the ${ }^{40} \mathrm{~K}$ emissions from the $\mathrm{NaI}(\mathrm{Tl})$ crystals) $[63,64]$; the data taking started in September 2016 with an energy threshold of $2 \mathrm{keV}_{e e}$. From the analysis of the first 59.5 days of data collected, COSINE-100 excluded the DAMA/LIBRA signal as due to the SI interaction of WIMPs in a standard halo model [65]; these data have been also interpreted in other contexts like Inelastic Boosted DM [66] and WIMP effective models [67]. Results for solar axions have also been derived [68]. A first analysis of annual modulation from 1.7 years of data has been presented [69]; since three crystals were excluded (because of a reduced light yield), the total exposure analyzed is $97.79 \mathrm{~kg} \cdot \mathrm{y}$. The best fit for the modulation amplitude in the $2-6 \mathrm{keV}_{e e}$ gives $S_{m}=(0.0083 \pm 0.0068) \mathrm{c} / \mathrm{keV} / \mathrm{kg} / \mathrm{d}$. The COSINE-100 counting rate in the $1-6 \mathrm{keV}_{e e}$ region for the crystals considered has been 
evaluated as $2.7 \mathrm{c} / \mathrm{keV} / \mathrm{kg} / \mathrm{d}$ [70]. COSINE-100 is running and a $3 \sigma$ coverage of the DAMA/LIBRA region was foreseen for five years of data with full exposure. In parallel, in-house crystal manufacturing in order to reduce intrinsic radioactivity is ongoing in Korea for a new phase of the experiment using $200 \mathrm{~kg}$ of NaI(Tl) (COSINE-200) [71]; first results for small crystals (with a mass of $\sim 0.7 \mathrm{~kg}$ ) have shown a decrease of ${ }^{210} \mathrm{~Pb}$ activity [72].

The comparison between the modulation amplitudes obtained by DAMA/LIBRA, ANAIS-112 and COSINE-100 experiments is shown in Figure 1.

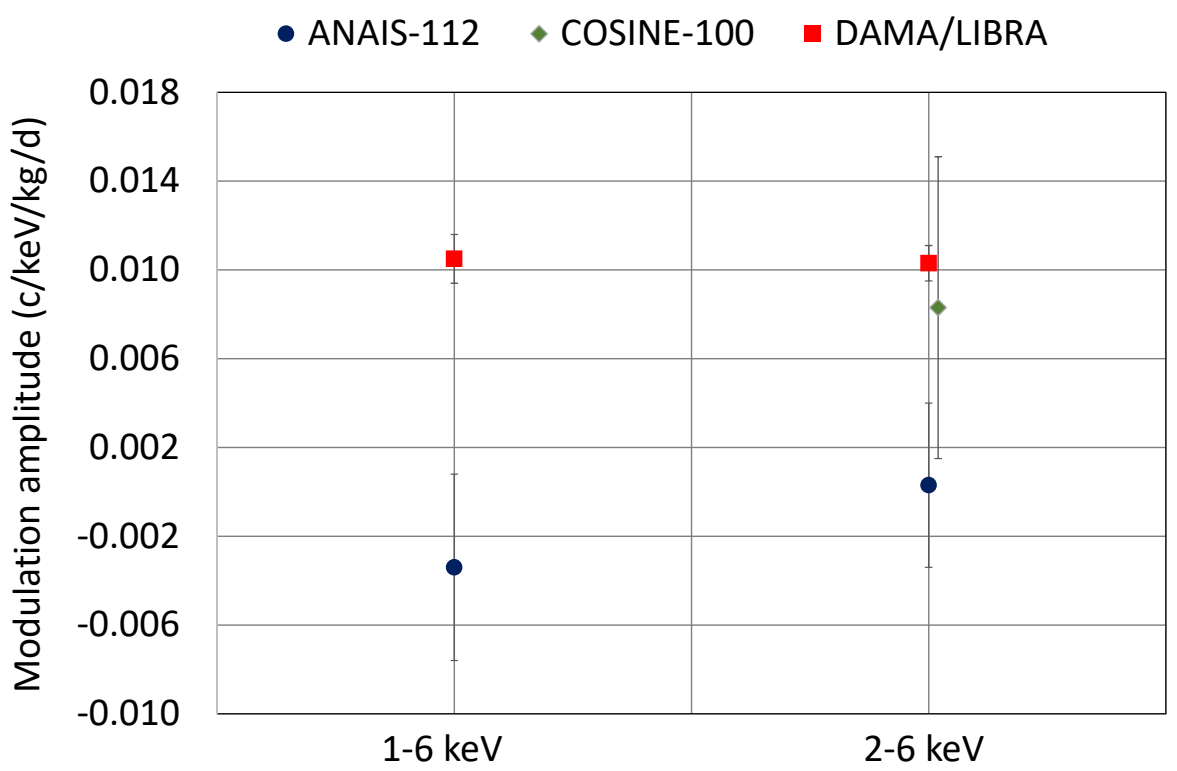

Figure 1. Comparison of the modulation amplitudes $S_{m}$ derived by DAMA/LIBRA [45], ANAIS112 [61] and COSINE-100 [69] experiments for the $1-6 \mathrm{keV}_{e e}$ and 2-6 $\mathrm{keV}_{e e}$ energy regions.

The SABRE project (Sodium-iodide with Active Background REjection) is being prepared at LNGS [73]; twin detectors in the northern and southern hemispheres (at LNGS and at the Stawell Laboratory, in construction in Australia, respectively) will be set-up in the end to analyze any possible seasonal effects induced by backgrounds, which would have opposite phase in those detectors. SABRE is working on the procurement of $\mathrm{NaI}(\mathrm{Tl})$ crystals with improved radiopurity; a potassium concentration of $(4.3 \pm 0.2) \mathrm{ppb}$ has been measured by ICPMS for a crystal [74] and further developments are in progress [75]. A target mass of $\sim 50 \mathrm{~kg}$ is planned to be operated using not only passive but also active shielding (with a veto made of liquid scintillator). A reduction of the background rate obtained by DAMA/LIBRA in the region of interest of about one order of magnitude is intended; Monte Carlo simulations for expected background have been made [76]. For an exposure of three years, DM-nucleon cross sections up to to $2 \times 10^{-42} \mathrm{~cm}^{2}$ could be explored for masses around $\sim 45 \mathrm{GeV} / \mathrm{c}^{2}$. Tests with a $3.5 \mathrm{~kg}$ crystal shipped from US to LNGS are ongoing (SABRE Proof of Principle, PoP).

The PICOLON (Pure Inorganic Crystal Observatory for LOw-energy Neutr(al)ino) project, placed at Kamioka, is also working on the development of $\mathrm{NaI}(\mathrm{Tl})$ detectors with ultra-high purity following recristallization and using appropriate resins, in order to reduce particularly ${ }^{210} \mathrm{~Pb}$ and ${ }^{40} \mathrm{~K}$, and satisfactory tests with small crystals have been made $[77,78]$. A first phase using four modules of $\mathrm{NaI}(\mathrm{Tl})$ with a total mass of $23.4 \mathrm{~kg}$ could be prepared in 2021 [78]. In a longer term and after completing various steps, the installation inside the KamLAND detector of a large mass (hundreds of $\mathrm{kg}$ ) of $\mathrm{NaI}(\mathrm{Tl})$ is being considered [79].

$\mathrm{NaI}(\mathrm{Tl})$ crystals were firstly considered as bolometers, operated at low temperatures, in [80]. The COSINUS experiment (Cryogenic Observatory for SIgnatures seen in Nextgeneration Underground Searches), located also at LNGS, is based on a different detection approach than the other $\mathrm{NaI}(\mathrm{Tl})$ projects, by developing scintillating bolometers made of 
NaI, following the CRESST technology [81]. The phonon signal does not depend on the type of particle generating the event but the scintillation light does, so a detector measuring simultaneously heat and light can discriminate NR and ER on an event-by-event basis. This capability has been shown with crystals having masses of tens of grams. Pure NaI could be used, thanks to the intrinsic scintillation at cryogenic temperatures. If COSINUS excluded a DM interaction rate of $\sim 0.01 \mathrm{cpd} / \mathrm{kg}$ above $1.8 \mathrm{keV}$, the DM interpretation of DAMA/LIBRA signal would be ruled out [82]. The construction of the experiment is underway and the first results on DM are expected for 2023.

Summarizing, several projects using sodium iodide as DM target are working to clarify the long-standing puzzle of the annual modulation observed over two decades by DAMA/LIBRA, being in conflict with exclusion results assuming certain scenarios from other experiments using different targets. ANAIS-112 and COSINE-100 have already presented very relevant results, although still with limited significance; the derived modulation amplitude is compatible with zero from 3 years of data of ANAIS-112, although from 1.7 years of data of COSINE-100 it is also compatible with that of DAMA/LIBRA. Other projects with particular, attractive features are also underway. The main features of all of them are summarized in Table 1.

Table 1. Summary of the main features of sodium iodide experiments and projects focused on the dark matter (DM) annual modulation signature.

\begin{tabular}{lclcccc}
\hline Experiment & Laboratory & Technology & Target & Size & Status & Reference \\
\hline DAMA/LIBRA & LNGS & Scintillator & $\mathrm{NaI}(\mathrm{Tl})$ & $\sim 250 \mathrm{~kg}$ & Running & {$[45,47]$} \\
ANAIS-112 & LSC & Scintillator & $\mathrm{NaI}(\mathrm{Tl})$ & $112.5 \mathrm{~kg}$ & Running & {$[59,60]$} \\
COSINE-100 & Yangyang & Scintillator & $\mathrm{NaI}(\mathrm{Tl})$ & $106 \mathrm{~kg}$ & Running & {$[69]$} \\
SABRE & LNGS,Stawell & Scintillator & $\mathrm{NaI}(\mathrm{Tl})$ & $\sim 50 \mathrm{~kg}$ & In preparation & {$[73]$} \\
PICOLON & Kamioka & Scintillator & $\mathrm{NaI}(\mathrm{Tl})$ & $23.4 \mathrm{~kg}$ & In preparation & {$[77]$} \\
COSINUS & LNGS & Bolometer & $\mathrm{NaI}, \mathrm{NaI}(\mathrm{Tl})$ & $\sim 1 \mathrm{~kg}$ & In preparation & {$[81]$} \\
\hline
\end{tabular}

\section{Signal Directionality}

As the Sun turns around the center of our galaxy, the wind of DM particles moving through the solar system is coming mainly from the Cygnus constellation. Then, the recording of the direction of tracks produced by NR could allow to demonstrate the galactic origin of a potential signal by discriminating DM events from backgrounds, expected to produce isotropic tracks [83-85]. It is estimated that the difference for the forwardbackward rates from DM interactions could be about a factor 10 (depending on the energy threshold). Moreover, after a detection of a DM signal, many properties of DM like their velocity distribution could be explored in this way, opening the possibility of the so-called DM astronomy. Directional DM experiments could explore cross sections below the limits set by neutrino interactions (neutrino floor), even having smaller exposures than other types of experiments, thanks to the different angular distributions of the recoils induced by DM or by neutrinos; atmospheric and supernova neutrinos would give roughly isotropic recoils, while those from solar neutrinos would concentrate in the opposite direction from the Sun. Moreover, directional detectors could be used not only for the direct detection of galactic DM particles but also to search for light DM behind an electron accelerator beam dump [86] and to detect supernova-produced DM (new particles with mass on the $\mathrm{MeV}$ scale with a flux steeply peaked towards the galactic center) [87].

The reconstruction of tracks in detectors is really challenging, since for NR with energies at the $\mathrm{keV}$ scale, paths are very short: $\sim 1 \mathrm{~mm}$ in gas media and $\sim 0.1 \mu \mathrm{m}$ in solid material. To fully determine the direction, it would be necessary to register axis and sense; but the observation of the so-called head-tail asymmetry, by registering the relative energy loss along the path, can be helpful. In addition, a daily modulation on the incoming direction of DM particles, due to the Earth's rotation, is also expected. 
Two approaches are being followed in the construction of directional detectors for DM. On one hand, using nuclear emulsions and, on the other, operating gas targets at low pressure $(\sim 0.1 \mathrm{~atm})$ inside TPCs, having different charge amplification systems and track readout devices, like MWPCs (Multi-Wire Proportional Chambers), MPGDs (Micro Pattern Gaseous Detectors) and optical readouts [88]. Low pressure gas targets are required to have longer tracks which could be more easily reconstructed. Then, the mass of the DM target accumulated in these experiments is typically much lower than in other DM detectors. Due to a different range and ionisation density, the discrimination between NR and ER is in some cases possible. It is worth noting that in most of the gas detectors, mixtures containing ${ }^{19} \mathrm{~F}$, with non-null spin, are used. In this section, the main projects working on all these approaches will be presented; Table 2 shows a summary of their main properties.

Table 2. Summary of the main features of projects focused on the directional DM detection.

\begin{tabular}{lcccccc}
\hline Experiment & Laboratory & Technology & Target & Size & Status & Reference \\
\hline DRIFT & Boulky & TPC+MWPC & $\mathrm{CS}_{2}+\mathrm{CF}_{4}+\mathrm{O}_{2}$ & $0.14 \mathrm{~kg}, 1 \mathrm{~m}^{3}$ & Finished & {$[89]$} \\
MIMAC & LSM & TPC+Micromegas & $\mathrm{CHF}_{3}+\mathrm{CF}_{4}+\mathrm{C}_{4} \mathrm{H}_{10}$ & $1 \mathrm{~m}^{3}$ & In preparation & {$[90,91]$} \\
NEWAGE & Kamioka & TPC+ $\mu$ PIC & $\mathrm{CF}_{4}, \mathrm{SF}_{6}$ & $0.01 \mathrm{~kg}$ & Running & {$[92,93]$} \\
DMTPC & WIPP & TPC+opt. read. & $\mathrm{CF}_{4}$ & $1 \mathrm{~m}^{3}$ & In preparation & {$[94]$} \\
CYGNO & LNGS & TPC+GEM,CMOS,PMT & $\mathrm{He} / \mathrm{CF}_{4}$ & $1 \mathrm{~m}^{3}$ & In preparation & {$[95,96]$} \\
\hline NEWS-dm & LNGS & Nuc. emulsion+opt. read & Silver halide & $10 \mathrm{~g}$ & In preparation & {$[97]$} \\
\hline
\end{tabular}

\subsection{Time Projection Chambers}

The DRIFT experiment (Directional Recoil Identification From Tracks) can be considered as the pioneer of directional DM detectors. It consisted of a TPC, having a conversion volume of around $1 \mathrm{~m}^{3}$, equipped with two attached MWPCs sharing a central cathode. The stainless steel vessel was filled with an electronegative gas, using a mixture made of $\mathrm{CS}_{2}+\mathrm{CF}_{4}+\mathrm{O}_{2}$ (corresponding to $0.14 \mathrm{~kg}$ at a pressure of $55 \mathrm{mbar}$ ); then, the produced ions, instead of the electrons, are drifted towards the readout, which helps to reduce the effects of diffusion and to optimize the resolution of tracks. This approach is scalable to large volumes, although the spatial granularity is limited (larger than $\sim 1 \mathrm{~mm}$ ). Background from ER can be rejected, but that from alpha particles is problematic. DRIFT operated at the Boulby Underground Laboratory, in UK, for more than ten years. Directional NR, induced by neutrons from a ${ }^{252} \mathrm{Cf}$ source, were measured and the head-tail asymmetry was quantified [98]. Constraints for SD DM-proton interaction were derived from an exposure of 54.7 days, down to $2.8 \times 10^{-37} \mathrm{~cm}^{2}$ for a mass of $100 \mathrm{GeV} / \mathrm{c}^{2}$ [89], which have recently been improved in the low mass region thanks to machine learning algorithms to better discriminate signal and background events [99].

The MIMAC experiment (MIcro-tpc MAtrix of Chambers) also uses a dual TPC with a shared cathode, which in this case is equipped with pixelized Micromegas (Micromesh Gas structures). The bulk Micromegas technology used offers stability and good spatial and energy resolution. MIMAC operates at the LSM since 2012. The stainless steel vessel is filled with a $\mathrm{CHF}_{3}+\mathrm{CF}_{4}+\mathrm{C}_{4} \mathrm{H}_{10}$ mixture, working at $\sim 50$ mbar. A low threshold of $2 \mathrm{keV}_{e e}$ has been achieved in a prototype. Quenching factors for $\mathrm{F}$ and He have been measured using directly an ion source at Grenoble. 3D tracks of NR from radon were also registered [90] and the observation with angular resolution lower than $10^{\circ}$ of ${ }^{19} \mathrm{~F}$ ion tracks (from an ion beam) has been achieved [91,100]. A detector with volume at the scale of $1 \mathrm{~m}^{3}$ is in preparation.

The NEWAGE experiment (NEw generation WIMP search with an Advanced Gaseous tracker Experiment) is based on a monolithic system with a TPC equipped with a micropixel ( $\mu$-PIC) chamber for amplification and readout. After operating on surface, long data-taking at the Kamioka underground laboratory was made filling the vessel with $\mathrm{CF}_{4}$ gas at $\sim 100$ mbar. NEWAGE also demonstrated the $3 \mathrm{D}$ detection of NR tracks, observed the head-tail effect at energies larger than $100 \mathrm{keV}$ and presented first results 
on DM interactions [92]. Limits for the SD DM-proton interaction cross section have been derived with data taken from 2013 to 2017 corresponding to $4.51 \mathrm{~kg} \cdot \mathrm{d}$, achieving a value of $4.3 \times 10^{2} \mathrm{pb}$ for WIMPs with mass of $150 \mathrm{GeV} / \mathrm{c}^{2}[93,101]$. Since 2018, a detector with low background specifications using polyimide is running [102]. Developments with $\mathrm{SF}_{6}$ gas in a negative ion micro-TPC detector are also underway [103].

The DMTPC experiment (Dark Matter Time-Projection Chamber) uses a TPC with both charge and optical (PMTs, CCDs) readouts providing complementary energy measurement; CCDs offer high granularity and quite easy data acquisition. Since 2007, various prototypes of 10 and 20 liters have been operated, firstly at MIT and later underground at WIPP (Waste Isolation Pilot Plant) facilities, in US. The vessel was filled with $\mathrm{CF}_{4}$ gas working at low pressure (30-100 Torr). The direction of NRs was measured in the 20 liter chamber [94]. Now, a detector at the $1 \mathrm{~m}^{3}$ scale is in preparation, using four TPCs.

CYGNUS is a proto-collaboration which has been set-up gathering many of the groups focused on directional DM detection; the aim is to develop a multitarget and multisite observatory of DM with capabilities to measure directionality [104]. Particular common goals are the study of different mixtures of gas, the reduction of the energy threshold below $1 \mathrm{keV}_{e e}$ in these type of detectors and the increase of the detector volumes (from 10 to even $1000 \mathrm{~m}^{3}$ ). Both high and low density gas targets can be considered, to work respectively either on search mode or with directionality. The science case for such a large NR observatory, a description of technologies and careful estimates of the expected sensitivity under different scenarios are presented in detail at [104]. There are projects underway to install CYGNUS detectors at several laboratories in US, UK, Japan, Italy and Australia and first prototypes are already being operated. For instance, CYGNO [95], at LNGS, has developed some small detectors using $\mathrm{He} / \mathrm{CF}_{4}$ and read by PMTs, CMOS cameras and GEMs [96,105]; NRs, produced by neutrons, registering direction and sense have been observed in the LEMOn (Large Elliptical Module Optically readout) prototype [106] and the achievement of a $2 \mathrm{keV}_{e e}$ energy threshold has been shown. The immediate goal is to build a $1 \mathrm{~m}^{3}$ apparatus, CYGNO/INITIUM, using the same technologies and with a target mass of $\sim 1 \mathrm{~kg}$.

\subsection{Other Techniques}

The NEWS-dm project (Nuclear Emulsions for WIMP Search with directional measurement) follows a different detection approach [97]. The target for DM and the tracking detector are made of an emulsion film having silver halide crystals immersed in a polymer; then, NRs generate silver clusters (at the nm scale) and the 3D tracks can be reconstructed using an optical microscope. NIT (Nano Imaging Tracker) emulsions using nanometric grains have been developed and new scanning systems, fully automated, to overcome diffraction limitations are being designed. The achievement of an excellent spatial resolution of $10 \mathrm{~nm}$ has been shown. Tests with a $10 \mathrm{~g}$ prototype are underway at LNGS in order to quantify backgrounds. The preservation of the direction of DM particles by NR has been studied by simulation using the SRIM code considering different recoil atom types finding a very good performance [107]. In a longer term, the operation of a detector put on an equatorial telescope (absorbing the rotation of the Earth) to follow the Cygnus constellation for $10 \mathrm{~kg} \cdot$ year has been proposed.

Together with all these efforts on directional detectors, other proposals have been made or are in development to determine the recoil direction in DM detectors, based on very different approaches:

- Following crystal defect spectroscopy, DM-induced NRs in a target made of diamond would produce an observable damage trail in the crystal altering the strain pattern [108,109]. An ultra-fine spatial resolution at the nm-scale is expected and, being a solid-state detector, a large target mass could be accumulated.

- $\quad$ A DNA strand detector could be implemented [110]; DNA strands prepared onto a nm-thick gold foil would be severed by the recoil of a gold atom, kicked out by a DM 
particle. The identification of the location of each severing event could be determined applying biological techniques.

- Planar targets like graphene make easier the determination of recoil directions as multiple interactions in the bulk target are avoided [111]. In PTOLEMY [112], graphene FETs in stacked planar arrays with tunable meV band gaps would offer single-electron sensitivity.

- In paleo-detectors, traces left in ancient minerals by the DM interaction could be searched for, taking advantage of huge integration times [113]. Different readout scenarios are being considered to achieve nm resolution and the mineral selection can be optimized to suppress some cosmogenic and radiogenic backgrounds.

- The dependence of the columnar recombination on the alignment of the NR direction with respect to a drift field provides a directional sensitivity, being investigated in the $\mathrm{ReD}$ (Recoil Directionality) project within the DarkSide Collaboration. This has been explored for a double-phase argon TPC [114].

- More ideas are being developed considering for instance carbon nanotubes [115] or anisotropic crystals like GaAs and sapphire [116] or scintillators like $\mathrm{ZnWO}_{4}$.

In summary, different projects for directional DM detection are underway all over the world exploiting different techniques. Due to the technological challenges these experiments must face, sensitivities worse than in other types of detector have been reached until now for exploring DM interaction cross sections and less stringent exclusion curves have been set for SI or SD couplings. But medium-size prototypes up to $1 \mathrm{~m}^{3}$ have been built or are in preparation, achieving important progress on fundamental requirements such as radiopurity, scalability or stability.

\section{Low-Mass DM}

Experiments intended to explore specifically potential light DM particles (below the $\mathrm{GeV} / \mathrm{c}^{2}$ scale) have additional requirements-lighter targets must be used to guarantee the kinematic matching between the DM particles and nuclei; lower energy thresholds are needed as even smaller signals are expected; and other new search channels have to be considered. As a light DM particle cannot transfer enough momentum to produce a detectable NR, scattering off not only by nuclei but also by electrons and absorption are being taken into account. It is worth noting that for low mass DM, the sensitivity is enhanced down to the $\mathrm{MeV} / \mathrm{c}^{2}$ scale when considering the Migdal effect [24]; it has been proposed that the DM-nucleus interaction could produce ionization or excitation of recoiling atoms (as electrons do not follow instantaneously the NR), which would give an additional ER signal particularly relevant for DM particles with low mass. The detection of bremsstrahlung emissions accompanying an undetectable NR is being also considered. It must be noted that the existence of these effects has still to be experimentally proven.

This need of extremely low energy thresholds and new detection channels has compelled over the last years the proposal and development of ultra-sensitive detection ideas based on advanced technologies to explore different mass ranges [16]. Experiments using ionization detectors, based on semiconductor devices or noble gas chambers, are producing very interesting results and will be mainly described here. Table 3 presents a summary of their main properties. 
Table 3. Summary of the main features of experiments and projects focused on low mass DM using purely ionization detectors.

\begin{tabular}{lcccccc}
\hline Experiment & Laboratory & Technology & Target & Size & Status & Reference \\
\hline CDEX-10 & Jinping & Point-Contact Ge & $\mathrm{Ge}$ & $\sim 10 \mathrm{~kg}$ & Running & {$[117,118]$} \\
DAMIC & SNOLAB & CCD & $\mathrm{Si}$ & $\sim 40 \mathrm{~g}$ & Running & {$[119]$} \\
DAMIC-M & LSM & Skipper CCD & $\mathrm{Si}$ & $\sim 700 \mathrm{~g}$ & In preparation & {$[120]$} \\
SENSEI & SNOLAB & Skipper CCD & $\mathrm{Si}$ & $100 \mathrm{~g}$ & In preparation & {$[121,122]$} \\
\hline SEDINE & LSM & Spherical Proportional Counter & $\mathrm{Ne}-\mathrm{CH}_{4}$ & $\sim 300 \mathrm{~g}$ & Finished & {$[123]$} \\
NEWS-G & SNOLAB & Spherical Proportional Counter & $\mathrm{H}, \mathrm{He}, \mathrm{Ne}$ & $\sim 1.4 \mathrm{~m}^{3}$ & In preparation & {$[124]$} \\
TREX-DM & LSC & TPC+Micromegas & $\mathrm{Ar} / \mathrm{Ne}$ & $300 / 160 \mathrm{~g}$ & In preparation & {$[125,126]$} \\
\hline
\end{tabular}

\subsection{Semiconductor Detectors}

As demonstrated by the CoGeNT experiment with a $440 \mathrm{~g}$ detector operated at the Soudan Underground Laboratory [127], Point-Contact germanium detectors can achieve sub-keV energy thresholds (due to the small capacitance) combined with excellent intrinsic radiopurity and a massive DM target, although the discrimination between ER and NR is nor possible. The CDEX experiment (China Dark matter EXperiment) is following this approach at the Jinping underground laboratory. CDEX-1 used two detectors with a mass of $\sim 1 \mathrm{~kg}$ each, with a threshold of $160 \mathrm{eV}_{e e}$. An annual modulation analysis (from 3.2 years of data and an energy threshold of $250 \mathrm{eV}_{e e}$ threshold) was made finding no signal [52] and limits on DM-nucleon SI and SD couplings, and considering the Migdal effect, were derived [117]. An array of $3 \times 3$ detectors immersed in liquid $\mathrm{N}_{2}$ with a total mass of $\sim 10 \mathrm{~kg}$ is used in CDEX-10; results for both SI and SD interactions from $102.8 \mathrm{~kg} \cdot \mathrm{d}$ data and an analysis threshold of $160 \mathrm{eV}_{e e}$ have been already released [118] as well as constraints on dark photons [128] and on WIMP couplings within the framework of effective field theories [129]. The preparation of larger set-ups (CDEX-100 and CDEX-1T) is underway with home-made germanium detectors to be placed inside a $1700 \mathrm{~m}^{3}$ liquid $\mathrm{N}_{2}$ tank. PointContact germanium detectors (n-type) are also used in the TEXONO project, which has set constraints on bosonic DM from $314.25 \mathrm{~kg} \cdot \mathrm{d}$ and upper limits on electron couplings [130].

In Charge-Coupled Devices (CCDs) made of silicon, the charge generated by the interaction drifts towards the pixel gates until being readout. As the interaction depth is correlated with the lateral charge diffusion, in this approach the 3D position can be reconstructed and effective particle identification is possible for background discrimination. The DAMIC experiment (DArk Matter In CCDs) operates 7 high resistivity, fully depleted CCDs ( $\sim 6$ g each) at SNOLAB since 2017; a leakage current at $2 \mathrm{e}^{-} / \mathrm{mm}^{2} /$ day has been achieved, with an energy threshold of $50 \mathrm{eV}_{e e}$. A precise determination of the quenching factor in Si down to $60 \mathrm{eV}_{e e}$ (using a Sb-Be source generating $24 \mathrm{keV}$ neutrons) was made and the radioactive impurities in silicon bulk were analyzed through time-correlated spatial coincidences [131]. Limits for low mass DM considering interactions on electrons and also hidden photon DM were obtained [132,133] and updated results on nucleon scattering from $11 \mathrm{~kg} \cdot \mathrm{d}$ have been presented [119]; above the threshold, an excess of charge events has been observed, which still requires investigation. DAMIC-M [120] will be operated at LSM using 50 more massive CCDs (with $13.5 \mathrm{~g}$ each) based on the Skipper readout, where through the multiple measurement of the pixel charges, noise is reduced and single electron counting at high resolution is achieved, as it has already been shown. It will be able to observe collision energies of only $1 \mathrm{eV}$. Commissioning of DAMIC-M in Modane is expected at 2023 and the goal is to achieve a background of $0.1 \mathrm{c} / \mathrm{keV} / \mathrm{kg} / \mathrm{d}$, thanks to the control of cosmogenic ${ }^{3} \mathrm{H}$ and surface ${ }^{210} \mathrm{~Pb}$. The cosmogenic activation of silicon has been carefully analyzed [134]. For a $1 \mathrm{~kg} \cdot \mathrm{y}$ exposure, DAMIC-M will explore SI DM-nucleon scattering at the $\mathrm{GeV} / \mathrm{c}^{2}$ range, DM-electron interactions from $1 \mathrm{MeV} / \mathrm{c}^{2}$ to $1 \mathrm{GeV} / \mathrm{c}^{2}$ and DM candidates from the hidden sector [120]. 
The novel Skipper readout is being already used by the SENSEI experiment (SubElectron-Noise Skipper CCD Experimental Instrument); a new generation of CCDs consisting of million pixels has been developed and small prototypes (with total active masses of 0.0947 and $2 \mathrm{~g}$ ) have been operated at Fermilab MINOS Hall in US (placed $100 \mathrm{~m}$ underground). SENSEI has set the most restrictive constraints on DM-electron scattering below a few $\mathrm{MeV} / \mathrm{c}^{2}[121,122]$ and a broad range of hidden-sector $\mathrm{DM}$ candidates can also be explored. The installation deep underground at SNOLAB of a 100-g detector (using 48 CCDs) with custom-designed electronics has been proposed. In a longer term, the Oscura project plans to operate $10 \mathrm{~kg}$ of Skipper CCDs.

\subsection{Gas Detectors}

A Spherical Proportional Counter (SPC) is an unconventional gas detector capable of reaching a very low energy threshold due to a reduced capacitance $(<1 \mathrm{pF})$ having a large volume [135]; a small ball at the sphere center acts as the anode, surrounded by the avalanche region. The SEDINE detector, operated at LSM, consisted of a sphere $(60 \mathrm{~cm}$ diameter) made of NOSV copper and filled with a gas mixture of $\mathrm{Ne}-\mathrm{CH}_{4}(0.7 \%)$ at a pressure of $3.1 \mathrm{bar}$ (giving an active mass of $\sim 300 \mathrm{~g}$ ). An acquisition energy threshold of $50 \mathrm{eV}_{e e}$ was achieved and exclusion results for SI DM interaction were derived from a set of data of 42 day [123]. The NEWS-G experiment (New Experiments With Spheres-Gas) will operate at SNOLab a larger sphere $(140 \mathrm{~cm}$ diameter) built in France and tested at LSM [124]. A thorough work has been made to mitigate ${ }^{210} \mathrm{~Pb}$ contamination in the copper sphere by electroplating a thin layer of ultra-radiopure copper onto the inner surface of the detector [136]. Determination of the quenching factors is underway for $\mathrm{H}$ from measurements at Grenoble and also at TUNL (Triangle Universities Nuclear Laboratory, Duke University) for $\mathrm{Ne}_{-} \mathrm{CH}_{4}(2 \%)$ down to $300 \mathrm{eV}_{e e}$. The response of the detector to single electrons (drift/diffusion times, gain, ...) was studied using a laser system [137] and tests of different sensors are ongoing $[138,139]$. Several light targets $(\mathrm{H}, \mathrm{He})$ are being considered and commissioning data with $\mathrm{CH}_{4}$ at 135 mbar (mass of $110 \mathrm{~g}$ ) were taken at LSM using the new large sphere before transportation to SNOLAB, where commissioning is ongoing. Thanks to the background reduction, better sensor performance and also improved analysis methods $[124,140]$, and considering $\mathrm{Ne}-\mathrm{CH}_{4}(10 \%)$, DM-nucleon cross sections down to $10^{-41} \mathrm{~cm}^{2}$ are expected to be explored for a mass of the DM particle of $1-2 \mathrm{GeV} / \mathrm{c}^{2}$. The development of electroformed copper spheres is being studied in collaboration with PNNL (Pacific Northwest National Laboratory); the design of ECUME, a $140 \mathrm{~cm}$ in diameter fully electroformed underground SPC, is underway and construction in SNOLAB is foreseen for 2021. In a longer term, the construction of a larger sphere ( $3 \mathrm{~m}$ diameter), DarkSPHERE, is being investigated; using $\mathrm{He}-\mathrm{C}_{4} \mathrm{H}_{10}(10 \%)$, the projected sensitivity reaches the neutrino floor in the sub-GeV mass range.

Other types of gas detectors are being developed too. The TREX-DM experiment (Tpcs for Rare Event eXperiments-Dark Matter) uses a gas TPC equipped with Micromegas readouts $[125,126,141]$, offering low intrinsic radioactivity and the possibility of recording topological information to discriminate backgrounds. The pressurized gas at 10 bar is held inside a vessel (with diameter and height of $0.5 \mathrm{~m}$ ) made of 6-cm-thick copper and the largest microbulk Micromegas planes ever built $\left(25 \times 25 \mathrm{~cm}^{2}\right)$ are being used. The detector is being presently commissioned at LSC. Being the target flexible, runs using Argon and Neon mixtures with isobutane have been performed below 8 bar; projections for the threshold range from $0.4 \mathrm{keV}_{e e}$ to $0.1 \mathrm{keV}_{e e}$. A complete background model has been made, predicting levels of a few $\mathrm{c} / \mathrm{keV} / \mathrm{kg} / \mathrm{d}$ in the region of interest [126].

\subsection{Other Techniques}

The stringent requirements to investigate low mass DM have pushed the development of novel technologies (proposed or already at R\&D phase) aimed at the recording of increasingly smaller energy deposits, the background suppression and the use of light targets: 
- In the HeRALD (Helium Roton Apparatus for Light Dark Matter) project, superfluid ${ }^{4} \mathrm{He}$ has been proposed as DM target [142]. Sensors consisting of TES (low temperature calorimeters) allow to measure quasiparticles and photons by quantum evaporation (liberation of ${ }^{4} \mathrm{He}$ atoms into a vacuum). Very low thresholds could be achieved as only $1 \mathrm{meV}$ is necessary to evaporate an He atom. In addition, He offers a light nuclear mass and copious production of scintillation light.

- In the SnowBall project, supercooled water (cooled below its normal freezing point) is proposed as DM target, offering the lightest target $(\mathrm{H})$ and the easy availability of water [143]. An interacting particle would trigger the water crystallization and a camera is used for the acquisition of image. Tests with neutrons using a $20 \mathrm{~g}$ prototype have been made operating at a temperature of $-20^{\circ}$, being insensitive to ER.

- Crystals made of laboratory-grown diamond acting as DM target could be outfitted with charge and phonon readouts to register the DM scattering (considering both NR and ER) of candidates having very low masses [144]. Carbon is lighter than other semiconductor materials and a sub-eV theshold can be expected thanks to low noise levels.

- Different small band gap materials (at 10-100 meV) from material informatics are being explored as sensors; the use of Si devices with Depleted P-channel Field Effect Transistor (DEPFET) could allow to reach sub-electron noise level to explore $\mathrm{MeV}$ DM particles [145]. Dirac materials with a small band gap of $\mathrm{O}(\mathrm{meV})$ could allow to explore even sub-MeV DM [146].

Shortly, purely ionization detectors are specially suited for the direct detection of low mass DM. The development of novel technologies for ionization detectors and sensors is ongoing but very low energy thresholds even below $0.1 \mathrm{keV}_{e e}$ have been already achieved in several experiments profiting from very low ionization energies or low capacitance. Although accumulating a large target mass is not easy in these type of detectors, nuclei with low mass number can be used, like $\mathrm{Si}$ in CCDs and $\mathrm{He}, \mathrm{Ne}$ or Ar in gas detectors. Then, relevant limits on DM-interaction cross sections have been set by these experiments, for nucleon scattering at masses below a few $\mathrm{GeV} / \mathrm{c}^{2}$ and, particularly, for electron scattering. In addition, other technologies are being explored in the path to detect the tiny energy deposits expected from very low mass DM particles.

\section{SD Interactions}

Bubble chambers operate using as targets metastable superheated liquids; then, following a local phase transition, dense energy depositions create bubbles which can be counted and localized with precision at $\mathrm{mm}$ by cameras. They are threshold detectors, as the recoil energy cannot be directly determined; dead times are large due to the required time to compress and decompress the detector after an interaction. However, these detectors are virtually unaffected by backgrounds producing ER as the bubble formation probability can be tuned so that only NR generate bubbles. Different target fluids can be used in bubble chambers, typically refrigerants; as most of them contain ${ }^{19} \mathrm{~F}$ (for instance $\mathrm{CF}_{3} \mathrm{I}$, $\mathrm{C}_{3} \mathrm{~F}_{8}$ or $\mathrm{C}_{4} \mathrm{~F}_{10}$ ), they have the highest sensitivity to $\mathrm{SD}$ interactions on protons. This type of detectors has been therefore focused on the study on SD DM couplings.

PICO joined together in 2012 the PICASSO and COUPP experiments and is operating since then a series of increasingly larger bubble chambers at SNOLAB. Following the first PICO-2L, in PICO-60, using $52 \mathrm{~kg}$ of $\mathrm{C}_{3} \mathrm{~F}_{8}$, an energy threshold of $2.45 \mathrm{keV}_{n r}$ was obtained and the present best limits from direct detection experiments have been derived on SD DM-proton interaction cross sections [147]. For PICO-40L, some changes have been implemented in the design, like the buffer-free concept using no water inside the inner vessel to suppress some backgrounds and with the chamber built "right-side-up" [148]; data taking with this detector is already starting. For the future, PICO-500 will be a tonnescale detector with sensitivity to explore proton interaction cross section of $10^{-42} \mathrm{~cm}^{2}$ for a mass of the DM particle of tens of $\mathrm{GeV} / \mathrm{c}^{2}$. 
Developments are underway to operate scintillating bubble chambers, combining the extreme electron rejection and simple instrumentation of a bubble chamber with the event-by-event energy resolution of a liquid scintillator like Argon or Xenon. The technique has been established for a $30 \mathrm{~g}$ xenon bubble chamber [149]. The SBC (Scintillating Bubble Chamber) project is preparing at Fermilab such a detector using a LAr chamber with $10 \mathrm{~kg}$, which could be operated at SNOLAB in 2023.

\section{Outlook and Summary}

In the quest of the DM particles which can be populating our galactic halo, one of the approaches followed is to register the scattering of those particles in a suitable detector. For this direct detection of DM, experiments based on noble liquids or solid-state cryogenic detectors, with increasing target mass and sophistication, have produced very relevant (even if negative) results for years. But complementary searches are developed based on other detection technologies; smaller scale experiments, focused on different physics cases, are indeed a very active field too.

The identification of distinctive signatures of DM interaction, like the annual modulation in the rates or the signal directionality, would help to confirm a DM detection. Relevant results have been presented by $\mathrm{NaI}(\mathrm{Tl})$ experiments, ANAIS-112 and COSINE-100, aimed to independently verify using the same target the DAMA/LIBRA annual modulation result observed over two decades; there are underway other $\mathrm{NaI}(\mathrm{Tl})$ projects too with attractive particular features. Although having higher background levels and energy threshold than other detectors, $\mathrm{NaI}(\mathrm{Tl})$ scintillators offer the robust and stable operation and the possibility of accumulating large target mass required in the investigation of the DM annual modulation signal.

The construction of a DM detector sensitive to directionality (and then capable of proving the galactic origin of a potential signal) is hard, as short tracks must be imaged with good resolution. Low-pressure TPCs equipped with different readouts (MWPCs, Micromegas, $\mu$ PICs, optical CCDs, ...) and nuclear emulsions are being implemented. Modest energy thresholds have been achieved in this type of detectors and collecting large exposures can be difficult, but medium-size TPC prototypes (having volumes from 0.1 to $1 \mathrm{~m}^{3}$ ) have already been developed and relevant progress is being achieved.

For SI DM-nucleon cross sections, the best limits for masses above a few $\mathrm{GeV} / \mathrm{c}^{2}$ come presently from XENON1T; but at lower masses and when considering SD interaction and also electron scattering, the leading results are obtained from several different detection technologies: solid-state cryogenic detectors (scintillating bolometers, Ge and Si crystals), liquid noble detectors (Xe, Ar) operated in S2-only mode (charge collection) but also purely ionization detectors (CCDs, Ge and gas detectors) and bubble chambers. Although in some cases having a large target mass is complicated, the achievement of extremely low energy thresholds, the inclusion of targets with low mass and/or the consideration of several interaction channels are the assets for exploring low mass DM; other new technologies are under study to further improve the detector performance.

It has been attempted to show here that small scale experiments have already released very competitive results in the direct detection of DM and, surely, more will come in the next future. Complementary experiments based on different technologies are required to guarantee the exploration of the wide range of possible DM candidate particles.

Funding: This research received no external funding.

Institutional Review Board Statement: Not applicable.

Informed Consent Statement: Not applicable.

Data Availability Statement: Not applicable.

Conflicts of Interest: The authors declare no conflict of interest. 


\section{References}

1. Bertone, G.; Hooper, D. History of dark matter. Rev. Mod. Phys. 2018, 90, 045002. [CrossRef]

2. Group, P.D.; Zyla, P.A.; Barnett, R.M.; Beringer, J.; Dahl, O.; Dwyer, D.A.; Groom, D.E.; Lin, C.J.; Lugovsky, K.S.; Pianori, E.; et al. Review of Particle Physics. Prog. Theor. Exp. Phys. 2020, 2020, 083C01. [CrossRef]

3. Liu, J.; Chen, X.; Ji, X. Current status of direct dark matter detection experiments. Nat. Phys. 2017, 13, 212. [CrossRef]

4. Conrad, J.; Reimer, O. Indirect dark matter searches in gamma and cosmic rays. Nat. Phys. 2017, 13, 224. [CrossRef]

5. Buchmueller, O.; Doglioni, C.; Wang, L.T. Search for dark matter at colliders. Nat. Phys. 2017, 13, 217. [CrossRef]

6. Fitzpatrick, A.L.; Haxton, W.; Katz, E.; Lubbers, N.; Xu, Y. The effective field theory of dark matter direct detection. JCAP 2013, 2013, 004. [CrossRef]

7. Drukier, A.K.; Freese, K.; Spergel, D.N. Detecting Cold Dark Matter Candidates. Phys. Rev. 1986, D33, 3495-3508. [CrossRef]

8. Review of mathematics, numerical factors, and corrections for dark matter experiments based on elastic nuclear recoil. Astropar. Phys. 1996, 6, 87. [CrossRef]

9. Schumann, M. Direct Detection of WIMP Dark Matter: Concepts and Status. J. Phys. G Nucl. Part Phys. 2019, 46, 103003. [CrossRef]

10. Heusser, G. Low-radioactivity background techniques. Ann. Rev. Nucl. Part Sci. 1995, 45, 543. [CrossRef]

11. Formaggio, J.; Martoff, C. Backgrounds to sensitive experiments underground. Ann. Rev. Nucl. Part Sci. 2004, 54, 361. [CrossRef]

12. Cebrián, S. Cosmogenic activation of materials. Int. J. Mod. Phys. A 2017, 32, 1743006. [CrossRef]

13. Cebrián, S. Cosmogenic activation in double beta decay experiments. Universe 2020, 6, 162. [CrossRef]

14. Billard, J.; Figueroa-Feliciano, E.; Strigari, L. Implication of neutrino backgrounds on the reach of next generation dark matter direct detection experiments. Phys. Rev. D 2014, 89, 023524. [CrossRef]

15. Marrodán-Undagoitia, T.; Rauch, L. Dark matter direct-detection experiments. J. Phys. G Nucl. Part Phys. 2015, $43,013001$. [CrossRef]

16. Battaglieri, M.; Belloni, A.; Chou, A.; Cushman, P.; Echenard, B.; Essig, R.; Estrada, J.; Feng, J.L.; Flaugher, B.; Fox, P.J.; et al. US Cosmic Visions: New Ideas in Dark Matter 2017, Community Report. arXiv 2017, arXiv:1707.04591.

17. Aprile, E.; Aalbers, J.; Agostini, F.; Alfonsi, M.; Althueser, L.; Amaro, F.; Anthony, M.; Arneodo, F.; Baudis, L.; Bauermeister, B.; et al. Dark Matter Search Results from a One Ton-Year Exposure of XENON1T. Phys. Rev. Lett. 2018, 121, 111302. [CrossRef]

18. Akerib, D.; Alsum, S.; Araújo, H.; Bai, X.; Bailey, A.; Balajthy, J.; Beltrame, P.; Bernard, E.; Bernstein, A.; Biesiadzinski, T.; et al. Results from a search for dark matter in the complete LUX exposure. Phys. Rev. Lett. 2017, 118, 021303. [CrossRef]

19. Cui, X.; Abdukerim, A.; Chen, W.; Chen, X.; Chen, Y.; Dong, B.; Fang, D.; Fu, C.; Giboni, K.; Giuliani, F.; et al. Dark Matter Results From 54-Ton-Day Exposure of PandaX-II Experiment. Phys. Rev. Lett. 2017, 119, 181302. [CrossRef] [PubMed]

20. Wang, Q.; Abdukerim, A.; Chen, W.; Chen, X.; Chen, Y.; Cheng, C.; Cui, X.; Fan, Y.; Fang, D.; Fu, C.; et al. Results of Dark Matter Search using the Full PandaX-II Exposure. arXiv 2020, arXiv:2007.15469.

21. Abe, K.; Hiraide, K.; Ichimura, K.; Kishimoto, Y.; Kobayashi, K.; Kobayashi, M.; Moriyama, S.; Nakahata, M.; Norita, T.; Ogawa, H.; et al. A direct dark matter search in XMASS-I. Phys. Lett. B 2019, 789, 45. [CrossRef]

22. Aprile, E.; Aalbers, J.; Agostini, F.; Alfonsi, M.; Althueser, L.; Amaro, F.; Antochi, V.; Angelino, E.; Arneodo, F.; Barge, D.; et al. Light Dark Matter Search with Ionization Signals in XENON1T. Phys. Rev. Lett. 2019, 123, 251801. [CrossRef]

23. Cheng, C.; Xie, P.; Abdukerim, A.; Chen, W.; Chen, X.; Chen, Y.; Cui, X.; Fan, Y.; Fang, D.; Fu, C.; et al. Search for Light Dark Matter-Electron Scatterings in the PandaX-II Experiment. arXiv 2021, arXiv:2101.07479.

24. Ibe, M.; Nakano, W.; Shoji, Y.; Suzuki, K. Migdal Effect in Dark Matter Direct Detection Experiments. JHEP 2018, $03,194$. [CrossRef]

25. Aprile, E.; Aalbers, J.; Agostini, F.; Alfonsi, M.; Althueser, L.; Amaro, F.; Antochi, V.; Angelino, E.; Arneodo, F.; Barge, D.; et al. Search for Light Dark Matter Interactions Enhanced by the Migdal effect or Bremsstrahlung in XENON1T. Phys. Rev. Lett. 2019, 123, 241803. [CrossRef] [PubMed]

26. Akerib, D.; Alsum, S.; Araújo, H.; Bai, X.; Balajthy, J.; Beltrame, P.; Bernard, E.; Bernstein, A.; Biesiadzinski, T.; Boulton, E.; et al. Results of a Search for Sub-GeV Dark Matter Using 2013 LUX Data. Phys. Rev. Lett. 2019, 122, 131301. [CrossRef] [PubMed]

27. Aprile, E.; Aalbers, J.; Agostini, F.; Alfonsi, M.; Althueser, L.; Amaro, F.; Antochi, V.; Angelino, E.; Angevaare, J.; Arneodo, F.; et al. Observation of Excess Electronic Recoil Events in XENON1T. Phys. Rev. D 2020, 102, 072004. [CrossRef]

28. Ajaj, R.; Amaudruz, P.; Araujo, G.; Baldwin, M.; Batygov, M.; Beltran, B.; Bina, C.; Bonatt, J.; Boulay, M.; Broerman, B.; et al. Search for dark matter with a 231-day exposure of liquid argon using DEAP-3600 at SNOLAB. Phys. Rev. D 2019, 100, 022004. [CrossRef]

29. Agnes, P.; Albuquerque, I.; Alexander, T.; Alton, A.; Araujo, G.; Ave, M.; Back, H.; Baldin, B.; Batignani, G.; Biery, K.; et al. DarkSide-50 532-day dark matter search with low-radioactivity argon. Phys. Rev. D 2018, 98, 102006. [CrossRef]

30. Agnes, P.; Albuquerque, I.; Alexander, T.; Alton, A.; Araujo, G.; Asner, D.; Ave, M.; Back, H.; Baldin, B.; Batignani, G.; et al. Low-Mass Dark Matter Search with the DarkSide-50 Experiment. Phys. Rev. Lett. 2018, 121, 081307. [CrossRef]

31. Agnes, P.; Albuquerque, I.; Alexander, T.; Alton, A.; Araujo, G.; Asner, D.; Ave, M.; Back, H.; Baldin, B.; Batignani, G.; et al. Constraints on Sub-GeV Dark-Matter-Electron Scattering from the DarkSide-50 Experiment. Phys. Rev. Lett. 2018, $121,111303$. [CrossRef]

32. Hehn, L.; Armengaud, E.; Arnaud, Q.; Augier, C.; Benoît, A.; Bergé, L.; Billard, J.; Blümer, J.; de Boissière, T.; Broniatowski, A.; et al. Improved EDELWEISS-III sensitivity for low-mass WIMPs using a profile likelihood approach. Eur. Phys. J. 2016, C76, 548. [CrossRef] 
33. Armengaud, E.; Augier, C.; Benoît, A.; Bergé, L.; Billard, J.; Broniatowski, A.; Camus, P.; Cazes, A.; Chapelier, M.; Charlieux, F.; et al. Searching for low-mass dark matter particles with a massive Ge bolometer operated above-ground. Phys. Rev. 2019, D99, 082003. [CrossRef]

34. Arnaud, Q.; Armengaud, E.; Augier, C.; Benoît, A.; Bergé, L.; Billard, J.; Broniatowski, A.; Camus, P.; Cazes, A.; Chapellier, M.; et al. First Germanium-Based Constraints on Sub-MeV Dark Matter with the EDELWEISS Experiment. Phys. Rev. Lett. 2020, 125, 141301. [CrossRef]

35. Agnese, R.; Aramaki, T.; Arnquist, I.; Baker, W.; Balakishiyeva, D.; Banik, S.; Barker, D.; Thakur, R.B.; Bauer, D.; et al. Results from the Super Cryogenic Dark Matter Search Experiment at Soudan. Phys. Rev. Lett. 2018, 120, 061802. [CrossRef]

36. Agnese, R.; Aralis, T.; Aramaki, T.; Arnquist, I.; Azadbakht, E.; Baker, W.; Banik, S.; Barker, D.; Bauer, D.; Binder, T.; et al. Search for Low-Mass Dark Matter with CDMSlite Using a Profile Likelihood Fit. Phys. Rev. 2019, D99, 062001. [CrossRef]

37. Amaral, D.; Aralis, T.; Aramaki, T.; Arnquist, I.; Azadbakht, E.; Banik, S.; Barker, D.; Bathurst, C.; Bauer, D.; Bezerra, L.; et al. Constraints on low-mass, relic dark matter candidates from a surface-operated SuperCDMS single-charge sensitive detector. Phys. Rev. D 2020, 102, 091101. [CrossRef]

38. Alkhatib, I.; Amaral, D.; Aralis, T.; Aramaki, T.; Arnquist, I.; Langroudy, I.A.; Azadbakht, E.; Banik, S.; Barker, D.; et al. Light Dark Matter Search with a High-Resolution Athermal Phonon Detector Operated Above Ground. arXiv 2020, arXiv:2007.14289.

39. Angloher, G.; Bento, A.; Bucci, C.; Canonica, L.; Defay, X.; Erb, A.; Feilitzsch, F.; Iachellini, N.F.; Gorla, P.; Gütlein, A.; et al. Results on light dark matter particles with a low-threshold CRESST-II detector. Eur. Phys. J. C 2016, 76, 25. [CrossRef]

40. Abdelhameed, A.H.; Angloher, G.; Bauer, P.; Bento, A.; Bertoldo, E.; Bucci, C.; Canonica, L.; D’Addabbo, A.; Defay, X.; Lorenzo, S.D.; et al. First results from the CRESST-III low-mass dark matter program. Phys. Rev. 2019, D100, 102002. [CrossRef]

41. Abdelhameed, A.; G. Angloher, P.B.; Bento, A.; Bertoldo, E.; Bucci, C.; Canonica, L.; D’ Addabbo, A.; Defay, X.; Lorenzo, S.D.; et al. First results on sub-GeV spin-dependent dark matter interactions with ${ }^{7} \mathrm{Li}$. Eur. Phys. J. C 2019, 79, 630. [CrossRef]

42. Freese, K.; Frieman, J.; Gould, A. Signal modulation in cold-dark-matter detection. Phys. Rev. D 1988, 37, 3388. [CrossRef]

43. Freese, K.; Lisanti, M.; Savage, C. Annual modulation of dark matter. Rev. Mod. Phys. 2013, 85, 1561. [CrossRef]

44. Bernabei, R.; Belli, P.; Cappella, F.; Cerulli, R.; Dai, C.; d’Angelo, A.; He, H.; Incicchitti, A.; Kuang, H.; Ma, J.; et al. First results from DAMA/LIBRA and the combined results with DAMA/NaI. Eur. Phys. J. C 2008, 56, 333-355. [CrossRef]

45. Bernabei, R.; Belli, P.; Bussolotti, A.; Cappella, F.; Caracciolo, V.; Cerulli, R.; Dai, C.; d'Angelo, A.; Marco, A.D.; He, H.; et al. First model independent results from DAMA/LIBRA-phase2. Nucl. Phys. Atom. Energy 2018, 19, 307. [CrossRef]

46. Bernabei, R.; Belli, P.; Cappella, F.; Caracciolo, V.; Castellano, S.; Cerulli, R.; Dai, C.; d'Angelo, A.; d'Angelo, S.; Marco, A.D.; et al. Final model independent result of DAMA/LIBRA-phase1. Eur. Phys. J. C 2013, 73, 2648. [CrossRef]

47. Bernabei, R.; Belli, P.; Bussolotti, A.; Cappella, F.; Caracciolo, V.; Cerulli, R.; Dai, C.; d'Angelo, A.; Marco, A.D.; Ferrari, N.; et al. The DAMA project: Achievements, implications and perspectives. Prog. Part Nucl. Phys. 2020, 114, 103810. [CrossRef]

48. Bernabei, R.; Belli, P.; Cappella, F.; Caracciolo, V.; Cerulli, R.; Dai, C.; d'Angelo, A.; Marco, A.D.; He, H.; Incicchitti, A.; et al. Improved model-dependent corollary analyses after the first six annual cycles of DAMA/LIBRA-phase2. Nucl. Phys. Atom. Energy 2019, 20, 317-348. [CrossRef]

49. Aprile, E.; Aalbers, J.; Agostini, F.; Alfonsi, M.; Amaro, F.; Anthony, M.; Arneodo, F.; Barrow, P.; Baudis, L.; Bauermeister, B.; et al. Search for Electronic Recoil Event Rate Modulation with 4 Years of XENON100 Data. Phys. Rev. Lett. 2017, 118, 101101. [CrossRef] [PubMed]

50. Akerib, D.; Alsum, S.; Araújo, H.; Bai, X.; Balajthy, J.; Beltrame, P.; Bernard, E.; Bernstein, A.; Biesiadzinski, T.; Boulton, E.; et al. Search for annual and diurnal rate modulations in the LUX experiment. Phys. Rev. 2018, D98, 062005. [CrossRef]

51. Kobayashi, M.; Abe, K.; Hiraide, K.; Ichimura, K.; Kishimoto, Y.; Kobayashi, K.; Moriyama, S.; Nakahata, M.; Ogawa, H.; Sato, K.; et al. Search for sub-GeV dark matter by annual modulation using XMASS-I detector. Phys. Lett. B 2019, 795, 308-313. [CrossRef]

52. Yang, L.; Li, H.; Yue, Q.; Ma, H.; Kang, K.; Li, Y.; Wong, H.; Agartioglu, M.; An, H.; Chang, J.; et al. Search for Light WeaklyInteracting-Massive-Particle Dark Matter by Annual Modulation Analysis with a Point-Contact Germanium Detector at the China Jinping Underground Laboratory. Phys. Rev. Lett. 2019, 123, 221301. [CrossRef]

53. Amaré, J.; Cebrián, S.; Coarasa, I.; Cuesta, C.; García, E.; Martínez, M.; Oliván, M.; Ortigoza, Y.; de Solórzano, A.O.; Puimedón, J.; et al. Performance of ANAIS-112 experiment after the first year of data taking. Eur. Phys. J. C 2019, 79, 228. [CrossRef]

54. Oliván, M.; Amaré, J.; Cebrián, S.; Cuesta, C.; García, E.; Martínez, M.; Ortigoza, Y.; Ortiz de Solórzano, A.; Pobes, C.; Puimedón, J.; et al. Light yield determination in large sodium iodide detectors applied in the search for dark matter. Astropart. Phys. 2017, 93, 86. [CrossRef]

55. Amaré, J.; Cebrián, S.; Cuesta, C.; García, E.; Ginestra, C.; Martínez, M.; Oliván, M.; Ortigoza, Y.; de Solórzano, A.O.; Pobes, C.; et al. Cosmogenic radionuclide production in NaI(Tl) crystals. JCAP 2015, 2015, 046. [CrossRef]

56. Amaré, J.; Cebrián, S.; Cuesta, C.; García, E.; Martínez, M.; Oliván, M.; Ortigoza, Y.; de Solórzano, A.O.; Puimedón, J.; Sarsa, M.; et al. Assessment of backgrounds of the ANAIS experiment for dark matter direct detection. Eur. Phys. J. C 2016, 76, 429. [CrossRef]

57. Amaré, J.; Cebrián, S.; Coarasa, I.; Cuesta, C.; García, E.; Martínez, M.; Oliván, M.; Ortigoza, Y.; de Solórzano, A.O.; Puimedón, J.; et al. Analysis of backgrounds for the ANAIS-112 dark matter experiment. Eur. Phys. J. C 2019, 79, 412. [CrossRef]

58. Coarasa, I.; Amaré, J.; Cebrián, S.; Cuesta, C.; García, E.; Martínez, M.; Oliván, M.; Ortigoza, Y.; de Solórzano, A.O.; Puimedón, J.; et al. ANAIS-112 sensitivity in the search for dark matter annual modulation. Eur. Phys. J. C 2019, 79, 233. [CrossRef] 
59. Amaré, J.; Cebrián, S.; Coarasa, I.; Cuesta, C.; García, E.; Martínez, M.; Oliván, M.; Ortigoza, Y.; de Solórzano, A.O.; Puimedón, J.; et al. First Results on Dark Matter Annual Modulation from the ANAIS-112 Experiment. Phys. Rev. Lett. 2019, $123,031301$. [CrossRef] [PubMed]

60. Amaré, J.; Cebrián, S.; Cintas, D.; Coarasa, I.; García, E.; Martínez, M.; Oliván, M.; Ortigoza, Y.; de Solórzano, A.O.; Puimedón, J.; et al. ANAIS-112 status: two years results on annual modulation. J. Phys. Conf. Ser. 2020, 1468, 012014. [CrossRef]

61. Amaré, J.; Cebrián, S.; Cintas, D.; Coarasa, I.; García, E.; Martínez, M.; Oliván, M.; Ortigoza, Y.; de Solórzano, A.O.; Puimedón, J.; et al. Annual Modulation Results from Three Years Exposure of ANAIS-112. arXiv 2021, arXiv:2103.01175.

62. Barbosa de Souza, E.; Cherwinka, J.; Cole, A.; Ezeribe, A.C.; Grant, D.; Halzen, F.; Heeger, K.M.; Hsu, L.; Hubbard, A.J.F.; et al. First search for a dark matter annual modulation signal with $\mathrm{NaI}(\mathrm{Tl})$ in the Southern Hemisphere by DM-Ice17. Phys. Rev. D 2017, 95, 032006. [CrossRef]

63. Adhikari, G.; Adhikari, P.; de Souza, E.B.; Carlin, N.; Choi, S.; Choi, W.; Djamal, M.; Ezeribe, A.; Ha, C.; Hahn, I.; et al. Initial Performance of the COSINE-100 Experiment. Eur. Phys. J. C 2018, 78, 107. [CrossRef]

64. Adhikari, P.; Adhikari, G.; de Souza, E.B.; Carlin, N.; Choi, S.; Choi, W.; Djamal, M.; Ezeribe, A.; Ha, C.; Hahn, I.; et al. Background model for the NaI(Tl) crystals in COSINE-100. Eur. Phys. J. C 2018, 78, 490. [CrossRef]

65. Adhikari, G.; Adhikari, P.; de Souza, E.B.; Carlin, N.; Choi, S.; Djamal, M.; Ezeribe, A.C.; Ha, C.H.; Hahn, I.; Hubbard, A.J.; et al. An experiment to search for dark matter interactions using sodium iodide detectors. Nature 2018, 564, 83. [CrossRef]

66. Ha, C.; Adhikari, G.; Adhikari, P.; Barbosa de Souza, E.; Carlin, N.; Choi, S.; Djamal, M.; Ezeribe, A.C.; Hahn, I.S.; Jeon, E.J.; et al. First Direct Search for Inelastic Boosted Dark Matter with COSINE-100. Phys. Rev. Lett. 2019, 122, 131802. [CrossRef]

67. Adhikari, G.; Adhikari, P.; de Souza, E.B.; Carlin, N.; Choi, S.; Djamal, M.; Ezeribe, A.; Ha, C.; Hahn, I.; Jeon, E.; et al. COSINE-100 and DAMA/LIBRA-phase2 in WIMP effective models. JCAP 2019, 06, 048. [CrossRef]

68. Adhikari, P.; Adhikari, G.; Barbosa de Souza, E.; Carlin, N.; Choi, S.; Djamal, M.; Ezeribe, A.; Ha, C.; Hahn, I.; Jeon, E.; et al. A search for solar axion induced signals with COSINE-100. Astropart. Phys. 2020, 114, 101. [CrossRef]

69. Adhikari, G.; Adhikari, P.; de Souza, E.B.; Carlin, N.; Choi, S.; Djamal, M.; Ezeribe, A.; Ha, C.; Hahn, I.; Jeon, E.; et al. Search for a Dark Matter-Induced Annual Modulation Signal in NaI(Tl) with the COSINE-100 Experiment. Phys. Rev. Lett. 2019, 123, 031302. [CrossRef]

70. Adhikari, G.; Adhikari, P.; de Souza, E.B.; Carlin, N.; Choi, J.; Choi, S.; Djamal, M.; Ezeribe, A.; Franca, L.; Ha, C.; et al. Background modeling for dark matter search with 1.7 years of COSINE-100 data. arXiv 2021, arXiv:2101.11377.

71. Shin, K.; Choe, J.; Gileva, O.; Iltis, A.; Kim, Y.; Lee, C.; Lee, H.; Lee, M.; Park, H. A facility for mass production of ultra-pure NaI powder for the COSINE-200 experiment. JINST 2020, 15, C07031. [CrossRef]

72. Park, B.; Choe, J.; Choi, J.; Gileva, O.; Ha, C.; Iltis, A.; Jeon, E.; Kim, D.; Kim, K.; Kim, S.; et al. Development of ultra-pure NaI(Tl) detectors for the COSINE-200 experiment. Eur. Phys. J. C 2020, 80, 814. [CrossRef]

73. Antonello, M.; Barberio, E.; Baroncelli, T.; Benziger, J.; Bignell, L.J.; Bolognino, I.; Calaprice, F.; Copello, S.; D’Angelo, D.; D'Imperio, G.; et al. The SABRE project and the SABRE Proof-of-Principle. Eur. Phys. J. C 2019, 79, 363. [CrossRef]

74. Suerfu, B.; Wada, M.; Peloso, W.; Souza, M.; Calaprice, F.; Tower, J.; Ciampi, G. Growth of Ultra-high Purity NaI(Tl) Crystal for Dark Matter Searches. Phys. Rev. Res. 2020, 2, 013223. [CrossRef]

75. Antonello, M.; Arnquist, I.J.; Barberio, E.; Baroncelli, T.; Benziger, J.; Bignell, L.; Bolognino, I.; Calaprice, F.; Copello, S.; Dafinei, I.; et al. Characterization of SABRE crystal NaI-33 with direct underground counting. arXiv 2020, arXiv:2012.02610.

76. Antonello, M.; Barberio, E.; Baroncelli, T.; Benziger, J.; Bignell, L.; Bolognino, I.; Calaprice, F.; Copello, S.; D’Angelo, D.; D’Imperio, G.; et al. Monte Carlo simulation of the SABRE PoP background. Astropart. Phys. 2019, 106, 1. [CrossRef]

77. Fushimi, K.I. Low Background Measurement by Means of NaI(Tl) Scintillator -Improvement of Sensitivity for Cosmic Dark Matter. Radioisotopes 2018, 67, 101. [CrossRef]

78. Fushimi, K.; Kanemitsu, Y.; Hirata, S.; Chernyak, D.; Hazama, R.; Ikeda, H.; Imagawa, K.; Ishiura, H.; Ito, H.; Kisimoto, T.; et al. Development of highly radiopure $\mathrm{NaI}(\mathrm{Tl})$ scintillator for PICOLON dark matter search project. arXiv 2021, arXiv:2101.00759.

79. Kozlov, A.; Chernyak, D.; Takemoto, Y.; Fushimi, K.; Imagawa, K.; Yasuda, K.; Ejiri, H.; Hazama, R.; Ikeda, H.; Inoue, K.; et al. Detectors for direct Dark Matter search at KamLAND. Nucl. Instrum. Meth. A 2020, 958, 162239. [CrossRef]

80. Coron, N.; Cuesta, C.; García, E.; Ginestra, C.; Girard, T.; Marcillac, P.; Martínez, M.; Ortigoza, Y.; de Solórzano, A.O.; Pobes, C.; et al. Study of parylene-coated $\mathrm{NaI}(\mathrm{Tl})$ at low temperatures for bolometric applications. Astropart. Phys. 2013, 47, 31. [CrossRef]

81. Angloher, G.; Hauff, D.; Gironi, L.; Gotti, C.; Pessina, G.; Gütlein, A.; Maino, M.; Nagorny, S.; Pagnanini, L.; Petricca, F.; et al. The COSINUS project-perspectives of a NaI scintillating calorimeter for dark matter search. Eur. Phys. J. C 2016, 76, 441. [CrossRef]

82. Kahlhoefer, F.; Reindl, F.; Schäffner, K.; Schmidt-Hoberg, K.; Wild, S. Model-independent comparison of annual modulation and total rate with direct detection experiments. JCAP 2018, 05, 074. [CrossRef]

83. Spergel, D.N. The Motion of the Earth and the Detection of Wimps. Phys. Rev. 1988, D37, 1353. [CrossRef]

84. Mayet, F.; Green, A.; Battat, J.; Billard, J.; Bozorgnia, N.; Gelmini, G.; Gondolo, P.; Kavanagh, B.; Lee, S.; Loomba, D.; et al. A review of the discovery reach of directional Dark Matter detection. Phys. Rep. 2016, 627, 1-49. [CrossRef]

85. Vahsen, S.; O’Hare, C.; Loomba, D. Directional recoil detection. arXiv 2021, arXiv:2102.04596.

86. Snowden-Ifft, D.P.; Harton, J.L.; Ma, N.; Schuckman, F.G. Directional light-WIMP time-projection-chamber detector for electron beam-dump experiments. Phys. Rev. D 2019, 99, 061301. [CrossRef] 
87. Baracchini, E.; DeRocco, W.; Dho, G. Discovering supernova-produced dark matter with directional detectors. Phys. Rev. D 2020, 102, 075036. [CrossRef]

88. Battat, J.; Irastorza, I.; Aleksandrov, A.; Guler, M.A.; Asada, T.; Baracchini, E.; Billard, J.; Bosson, G.; Bourrion, O.; Bouvier, J.; et al. Readout technologies for directional WIMP Dark Matter detection. Phys. Rep. 2016, 662, 1. [CrossRef]

89. Battat, J.; Ezeribe, A.; Gauvreau, J.; Harton, J.; Lafler, R.; Law, E.; Lee, E.; Loomba, D.; Lumnah, A.; Miller, E.; et al. Low Threshold Results and Limits from the DRIFT Directional Dark Matter Detector. Astropart. Phys. 2017, 91, 65. [CrossRef]

90. Riffard, Q.; Santos, D.; Guillaudin, O.; Bosson, G.; Bourrion, O.; Bouvier, J.; Descombes, T.; Fourel, C.; Muraz, J.F.; Lebreton, L.; et al. First detection of radon progeny recoil tracks by MIMAC. JINST 2017, 12, P06021. [CrossRef]

91. Tao, Y.; Beaufort, C.; Moric, I.; Tao, C.; Santos, D.; Sauzet, N.; Couturier, C.; Guillaudin, O.; Muraz, J.; Naraghi, F.; et al. Track length measurement of ${ }^{19} \mathrm{~F}^{+}$ions with the MIMAC directional Dark Matter detector prototype. Nucl. Instrum. Meth. A 2021, 985, 164569. [CrossRef]

92. Nakamura, K.; Miuchi, K.; Tanimori, T.; Kubo, H.; Takada, A.; Parker, J.; Mizumoto, T.; Mizumura, Y.; Nishimura, H.; Sekiya, H.; et al. Direction-sensitive dark matter search with gaseous tracking detector NEWAGE-0.3b'. Prog. Theor. Exp. Phys. 2015, 2015, 43F01. [CrossRef]

93. Yakabe, R.; Nakamura, K.; Ikeda, T.; Ito, H.; Yamaguchi, Y.; Taishaku, R.; Nakazawa, M.; Ishiura, H.; Nakamura, T.; Shimada, T.; et al. First limits from a 3d-vector directional dark matter search with the NEWAGE-0.3b' detector. arXiv 2020, arXiv:2005.05157.

94. Deaconu, C.; Leyton, M.; Corliss, R.; Druitt, G.; Eggleston, R.; Guerrero, N.; Henderson, S.; Lopez, J.; Monroe, J.; Fisher, P. Measurement of the directional sensitivity of DMTPC detectors. Phys. Rev. D 2017, 95, 122002. [CrossRef]

95. Baracchini, E.; Benussi, L.; Bianco, S.; Capoccia, C.; Caponero, M.; Cavoto, G.; Cortez, A.; Costa, I.A.; Marco, E.D.; D’Imperio, G.; et al. CYGNO: A gaseous TPC with optical readout for dark matter directional search. J. Instrum. 2020, 15, C07036. [CrossRef]

96. Costa, I.A.; Baracchini, E.; Bellini, F.; Benussi, L.; Bianco, S.; Caponero, M.; Cavoto, G.; D'Imperio, G.; Marco, E.D.; Maccarrone, G.; et al. Performance of optically readout GEM-based TPC with a 55Fe source. J. Instrum. 2019, 14, P07011. [CrossRef]

97. Agafonova, N.; Aleksandrov, A.; Anokhina, A.; Asada, T.; Ashikhmin, V.; Bodnarchuk, I.; Buonaura, A.; Chernyavskii, M.; Chukanov, A.; D'Ambrosio, N.; et al. Discovery potential for directional Dark Matter detection with nuclear emulsions. Eur. Phys. J. C 2018, 78, 578. [CrossRef]

98. Battat, J.; Daw, E.; Ezeribe, A.; Gauvreau, J.L.; Harton, J.L.; Lafler, R.; Lee, E.R.; Loomba, D.; Lumnah, A.; Miller, E.; et al. First measurement of nuclear recoil head-tail sense in a fiducialised WIMP dark matter detector. J. Instrum. 2016, 11, P10019. [CrossRef]

99. Battat, J.; Eldridge, C.; Ezeribe, A.; Gaunt, O.; Gauvreau, J.L.; Gregorio, R.M.; Habich, E.; Hall, K.; Harton, J.; Ingabire, I.; et al. Improved Sensitivity of the DRIFT-IId Directional Dark Matter Experiment using Machine Learning. arXiv 2021, arXiv:2103.06702.

100. Tao, Y.; Beaufort, C.; Moric, I.; Tao, C.; Santos, D.; Sauzet, N.; Couturier, C.; Guillaudin, O.; Muraz, J.; Naraghi, F.; et al. Dark Matter Directionality Detection performance of the Micromegas-based $\mu$ TPC-MIMAC detector. arXiv 2020, arXiv:2003.11812.

101. Ikeda, T.; Nakamura, K.; Shimada, T.; Yakabe, R.; Hashimoto, T.; Ishiura, H.; Nakamura, T.; Ito, H.; Ichimura, K.; Abe, K.; et al. Direction-sensitive dark matter search with a low-background gaseous detector NEWAGE-0.3b. arXiv 2021, arXiv:2101.09921.

102. Hashimoto, T.; Miuchi, K.; Ikeda, T.; Ishiura, H.; Nakamura, K.D.; Ito, H.; Ichimura, K.; Abe, K.; Kobayashi, K.; Takada, A.; et al. Development of a low- $\alpha$-emitting $\mu$-PIC as a readout device for direction-sensitive dark matter detectors. Nucl. Instrum. Meth. A 2020, 977, 164285. [CrossRef]

103. Ikeda, T.; Shimada, T.; Ishiura, H.; Nakamura, K.; Nakamura, T.; Miuchi, K. Development of a Negative Ion Micro TPC Detector with $\mathrm{SF}_{6}$ Gas for the Directional Dark Matter Search. JINST 2020, 15, P07015. [CrossRef]

104. Vahsen, S.; O’Hare, C.; Lynch, W.; Spooner, N.; Baracchini, E.; Barbeau, P.; Battat, J.; Crow, B.; Deaconu, C.; Eldridge, C.; et al. CYGNUS: Feasibility of a nuclear recoil observatory with directional sensitivity to dark matter and neutrinos. arXiv 2020, arXiv:2008.12587.

105. Baracchini, E.; Benussi, L.; Bianco, S.; Capoccia, C.; Caponero, M.; Cavoto, G.; Cortez, A.; Costa, I.A.; Marco, E.D.; D’Imperio, G.; et al. Stability and detection performance of a GEM-based Optical Readout TPC with $\mathrm{He} / \mathrm{CF}_{4}$ gas mixtures. JINST 2020, 15, P10001. [CrossRef]

106. Baracchini, E.; Benussi, L.; Bianco, S.; Capoccia, C.; Caponero, M.A.; Cavoto, G.; Cortez, A.; Costa, I.A.; Marco, E.D.; D’Imperio, G.; et al. Identification of low energy nuclear recoils in a gas TPC with optical readout. Meas. Sci. Tech. 2021, $32,025902$. [CrossRef]

107. Alexandrov, A.; Lellis, G.D.; Crescenzo, A.D.; Golovatiuk, A.; Tioukov, V. Directionality preservation of nuclear recoils in an emulsion detector for directional dark matter search. arXiv 2021, arXiv:2102.03125.

108. Rajendran, S.; Zobrist, N.; Sushkov, A.O.; Walsworth, R.; Lukin, M. A method for directional detection of dark matter using spectroscopy of crystal defects. Phys. Rev. D 2017, 96, 035009. [CrossRef]

109. Marshall, M.C.; Turner, M.J.; Ku, M.J.; Phillips, D.F.; Walsworth, R.L. Directional detection of dark matter with diamond. arXiv 2020, arXiv:2009.01028.

110. Drukier, A.K.; Cantor, C.; Chonofsky, M.; Church, G.; Fagaly, R.; Freese, K.; Lopez, A.; Sano, T.; Savage, C.; Wong, W.P. New class of biological detectors for WIMPs. Int. J. Mod. Phys. A 2014, 29, 1443007. [CrossRef]

111. Wang, S.Y. Graphene-based detectors for directional dark matter detection. Eur. Phys. J. C 2019, 79, 561. [CrossRef]

112. Baracchini, E.; Betti, M.; Biasotti, M.; Bosca, A.; Calle, F.; Carabe-Lopez, J.; Cavoto, G.; Chang, C.; Cocco, A. PTOLEMY: A Proposal for Thermal Relic Detection of Massive Neutrinos and Directional Detection of MeV Dark Matter. arXiv 2018, arXiv:1808.01892. 
113. Drukier, A.K.; Baum, S.; Freese, K.; Górski, M.; Stengel, P. Paleo-detectors: Searching for dark matter with ancient minerals. Phys. Rev. D 2019, 99, 043014. [CrossRef]

114. Cadeddu, M.; Lissia, M.; Agnes, P.; Batignani, G.; Bonivento, W.; Bottino, B.; Caravati, M.; Catalanotti, S.; Cataudella, V.; Cicalò, C.; et al. Directional dark matter detection sensitivity of a two-phase liquid argon detector. JCAP 2019, 01, 014. [CrossRef]

115. Cavoto, G.; Cirillo, E.; Cocina, F.; Ferretti, J.; Polosa, A. WIMP detection and slow ion dynamics in carbon nanotube arrays. Eur. Phys. J. C 2016, 76, 349. [CrossRef] [PubMed]

116. Griffin, S.; Knapen, S.; Lin, T.; Zurek, K.M. Directional detection of light dark matter with polar materials. Phys. Rev. D 2018, 98, 115034. [CrossRef]

117. Liu, Z.; Yue, Q.; Yang, L.; Kang, K.; Li, Y.; Wong, H.; Agartioglu, M.; An, H.; Chang, J.; Chen, J.; et al. Constraints on SpinIndependent Nucleus Scattering with sub-GeV Weakly Interacting Massive Particle Dark Matter from the CDEX-1B Experiment at the China Jinping Underground Laboratory. Phys. Rev. Lett. 2019, 123, 161301. [CrossRef]

118. Jiang, H.; Jia, L.; Yue, Q.; Kang, K.; Cheng, J.; Li, Y.; Wong, H.; Agartioglu, M.; An, H.; Chang, J.; et al. Limits on Light Weakly Interacting Massive Particles from the First $102.8 \mathrm{~kg} \times$ day Data of the CDEX-10 Experiment. Phys. Rev. Lett. 2018, 120, 241301. [CrossRef] [PubMed]

119. Aguilar-Arevalo, A.; Amidei, D.; Baxter, D.; Cancelo, G.; Vergara, B.C.; Chavarria, A.; D’Olivo, J.; Estrada, J.; Favela-Perez, F.; Gaior, R.; et al. Results on Low-Mass Weakly Interacting Massive Particles from an $11 \mathrm{~kg}$ d Target Exposure of DAMIC at SNOLAB. Phys. Rev. Lett. 2020, 125, 241803. [CrossRef]

120. Castello-Mor, N. DAMIC-M experiment: Thick, silicon CCDs to search for light dark matter. Nucl. Instrum. Meth. A 2019, 958, 162933. [CrossRef]

121. Abramoff, O.; Barak, L.; Bloch, I.M.; Chaplinsky, L.; Crisler, M.; Dawa.; Drlica-Wagner, A.; Essig, R.; Estrada, J.; Etzion, E.; Fernandez, G.; et al. SENSEI: Direct-Detection Constraints on Sub-GeV Dark Matter from a Shallow Underground Run Using a Prototype Skipper-CCD. Phys. Rev. Lett. 2019, 122, 161801. [CrossRef] [PubMed]

122. Barak, L.; Bloch, I.M.; Cababie, M.; Cancelo, G.; Chaplinsky, L.; Chierchie, F.; Crisler, M.; Drlica-Wagner, A.; Essig, R.; Estrada, J.; et al. SENSEI: Direct-Detection Results on sub-GeV Dark Matter from a New Skipper CCD. Phys. Rev. Lett. 2020, 125, 171802. [CrossRef] [PubMed]

123. Arnaud, Q.; Asner, D.; Bard, J.; Brossard, A.; Cai, B.; Chapellier, M.; Clark, M.; Corcoran, E.; Dandl, T.; Dastgheibi-Fard, A.; et al. First results from the NEWS-G direct dark matter search experiment at the LSM. Astropart. Phys. 2018, 97, 54. [CrossRef]

124. Giroux, G.; Gros, P.; Katsioulas, I. The search for light dark matter with the NEWS-G spherical proportional counter. J. Phys. Conf. Ser. 2019, 1312, 012008. [CrossRef]

125. Iguaz, F.; Garza, J.; Aznar, F.; Castel, J.; Cebrián, S.; Dafni, T.; García, J.; Irastorza, I.; Lagraba, A.; Luzón, G.; Peiró, A. TREX-DM: A low-background Micromegas-based TPC for low-mass WIMP detection. Eur. Phys. J. 2016, C76, 529. [CrossRef]

126. Castel, J.; Cebrian, S.; Coarasa, I.; Dafni, T.; Galan, J.; Iguaz, F.; Irastorza, I.; Luzon, G.; Mirallas, H.; de Solorzano, A.O.; Ruiz-Choliz, E. Background assessment for the TREX dark matter experiment. Eur. Phys. J. C 2019, 79, 782. [CrossRef]

127. Aalseth, C.E.; Barbeau, P.; Colaresi, J.; Collar, J.; Leon, J.D.; Fast, J.; Fields, N.; Hossbach, T.W.; Knecht, A.; Kos, M.; et al. CoGeNT: A Search for Low-Mass Dark Matter using p-type Point Contact Germanium Detectors. Phys. Rev. D 2013, 88, 012002. [CrossRef]

128. She, Z.; Jia, L.P.; Yue, Q.; Ma, H.; Kang, K.J.; Li, Y.J.; Agartioglu, M.; An, H.P.; Chang, J.P.; Chen, J.H.; et al. Direct Detection Constraints on Dark Photons with the CDEX-10 Experiment at the China Jinping Underground Laboratory. Phys. Rev. Lett. 2020, 124, 111301. [CrossRef]

129. Wang, Y.; Zeng, Z.; Yue, Q.; Yang, L.; Kang, K.; Li, Y.; Agartioglu, M.; An, H.; Chang, J.; Chen, J.; et al. First Experimental Constraints on WIMP Couplings in Effective Field Theory Framework from the CDEX Experiment. arXiv 2020, arXiv:2007.15555.

130. Singh, M.K.; Singh, L.; Agartioglu, M.; Sharma, V.; Singh, V.; Wong, H.T. Constraints on bosonic dark matter with low threshold germanium detector at Kuo-Sheng reactor neutrino laboratory. Chin. J. Phys. 2019, 58, 63. [CrossRef]

131. Aguilar-Arevalo, A.; Amidei, D.; Baxter, D.; Cancelo, G.; Vergara, B.C.; Chavarria, A.; Darragh-Ford, E.; D’Olivo, J.; Estrada, J.; Favela-Perez, F.; et al. Measurement of the bulk radioactive contamination of detector-grade silicon with DAMIC at SNOLAB. arXiv 2011, arXiv:2011.12922.

132. Aguilar-Arevalo, A.; Amidei, D.; Bertou, X.; Butner, M.; Cancelo, G.; Vázquez, A.C.; Vergara, B.C.; Chavarria, A.; Chavez, C.; de Mello Neto, J.; et al. First Direct-Detection Constraints on eV-Scale Hidden-Photon Dark Matter with DAMIC at SNOLAB. Phys. Rev. Lett. 2017, 118, 141803. [CrossRef]

133. Aguilar-Arevalo, A.; Amidei, D.; Baxter, D.; Cancelo, G.; Cervantes Vergara, B.A.; Chavarria, A.E.; Darragh-Ford, E.; de Mello Neto, J.R.T.; D'Olivo, J.C.; Estrada, J.; et al. Constraints on Light Dark Matter Particles Interacting with Electrons from DAMIC at SNOLAB. Phys. Rev. Lett. 2019, 123, 181802. [CrossRef]

134. Saldanha, R.; Thomas, R.; Tsang, R.H.M.; Chavarria, A.E.; Bunker, R.; Burnett, J.L.; Elliott, S.R.; Matalon, A.; Mitra, P.; Piers, A.; et al. Cosmogenic activation of silicon. Phys. Rev. D 2020, 102, 102006. [CrossRef]

135. Giomataris, I.; Irastorza, I.; Savvidis, I.; Andriamonje, S.; Aune, S.; Chapelier, M.; Charvin, P.; Colas, P.; Derre, J.; Ferrer, E.; et al. A Novel large-volume Spherical Detector with Proportional Amplification read-out. JINST 2008, 03, P09007. [CrossRef]

136. Balogh, L.; Beaufort, C.; Brossard, A.; Bunker, R.; Caron, J.F.; Chapellier, M.; Coquillat, J.M.; Corcoran, E.; Crawford, S.; Dastgheibi Fard, A.; et al. Copper electroplating for background suppression in the NEWS-G experiment. Nucl. Instrum. Meth. A 2021, 988, 164844. [CrossRef] 
137. Arnaud, Q.; Bard, J.; Brossard, A.; Chapellier, M.; Clark, M.; Crawford, S.; Corcoran, E.; Dastgheibi-Fard, A.; Dering, K.; Stefano, P.D.; et al. Precision laser-based measurements of the single electron response of SPCs for the NEWS-G light dark matter search experiment. Phys. Rev. D 2019, 99, 102003. [CrossRef]

138. Katsioulas, I.; Giomataris, I.; Knights, P.; Gros, M.; Navick, X.F.; Nikolopoulos, K.; Savvidis, I. A sparkless resistive glass correction electrode for the spherical proportional counter. JINST 2018, 13, P11006. [CrossRef]

139. Giomataris, I.; Gros, M.; Katsioulas, I.; Knights, P.; Mols, J.; Neep, T.; Nikolopoulos, K.; Savvidis, G.; Savvidis, I.; Shang, L.; et al. A resistive ACHINOS multi-anode structure with DLC coating for spherical proportional counters. JINST 2020, 15, 11. [CrossRef]

140. Katsioulas, I.; Knights, P.; Matthews, J.; Neep, T.; Nikolopoulos, K.; Owen, R.; Ward, R. Development of a simulation framework for spherical proportional counters. JINST 2020, 15, C06013. [CrossRef]

141. Irastorza, I.G.; Aznar, F.; Castel, J.; Cebrián, S.; Dafni, T.; Galán, J.; García, J.A.; Garza, J.G.; Gómez, H.; Herrera, D.; Iguaz, F.; et al. Gaseous time projection chambers for rare event detection: Results from the T-REX project. II. Dark matter. arXiv 2016, arXiv:1512.06294.

142. Hertel, S.A.; Biekert, A.; Lin, J.; Velan, V.; McKinsey, D.N. Direct detection of sub-GeV dark matter using a superfluid ${ }^{4}$ He target. Phys. Rev. D 2019, 100, 092007. [CrossRef]

143. Szydagis, M.; Huang, Y.; Kamaha, A.C.; Knight, C.C.; Levy, C.; Rischbieter, G.R. Evidence for the Freezing of Supercooled Water by Means of Neutron Irradiation. arXiv 2018, arXiv:1807.09253.

144. Kurinsky, N.; Yu, T.C.; Hochberg, Y.; Cabrera, B. Diamond detectors for direct detection of sub-GeV dark matter. Phys. Rev. D 2019, 99, 123005. [CrossRef]

145. Bähr, A.; Kluck, H.; Ninkovic, J.; Schieck, J.; Treis, J. DEPFET detectors for direct detection of MeV Dark Matter particles. Eur. Phys. J. C 2017, 77, 905. [CrossRef]

146. Hochberg, Y.; Kahn, Y.; Lisanti, M.; Zurek, K.M.; Grushin, A.G.; Ilan, R.; Griffin, S.M.; Liu, Z.F.; Weber, S.F.; Neaton, J.B. Detection of sub-MeV dark matter with three-dimensional Dirac materials. Phys. Rev. D 2018, 97, 015004. [CrossRef]

147. Amole, C.; Ardid, M.; Arnquist, I.J.; Asner, D.M.; Baxter, D.; Behnke, E.; Bressler, M.; Broerman, B.; Cao, G.; Chen, C.; et al. Dark Matter Search Results from the Complete Exposure of the PICO-60 C $\mathrm{F}_{8}$ Bubble Chamber. Phys. Rev. D 2019, $100,022001$. [CrossRef]

148. Bressler, M.; Campion, P.; Cushman, V.S.; Morrese, A.; Wagner, J.M.; Zerbo, S.; Neilson, R.; Crisler, M.; Dahl, C.E. A buffer-free concept bubble chamber for PICO dark matter searches. JINST 2019, 15, P08019. [CrossRef]

149. Baxter, D. First demonstration of a Scintillating Xenon Bubble Chamber for Detecting Dark Matter and Coherent Elastic Neutrino-Nucleus Scattering. Phys. Rev. Lett. 2017, 118, 231301. [CrossRef] 